\title{
Prozessorientierte Planung und Kalkulation (kern-)produktbegleitender Dienstleistungen
}

\author{
Klaus Möller · Ingo Cassack
}

Published online: 14 June 2008

(C) The Author(s) 2008

Zusammenfassung Die Bedeutung von Dienstleistungen nimmt volks- und betriebswirtschaftlich immer weiter zu. Die zunehmende Nachfrage führt jedoch in vielen Fällen dazu, dass die Dienstleistungen wenig systematisch und unreflektiert auf Nachfrage durch Kunden angeboten werden. Eine systematische Planung, Entwicklung und Kalkulation finden - im deutlichen Gegensatz zum physischen Produkt - nicht statt. Allerdings wird ein solches Vorgehen auch durch verschiedene Spezifika der Dienstleistungen erschwert. Der vorliegende Beitrag konzentriert sich auf (kern-) produktbegleitende Dienstleistungen und zeigt zunächst wesentliche theoretische Grundlagen einer prozessorientierten Planung und Kalkulation auf. Basis stellt dabei eine Weiterentwicklung der Prozesskostenrechnung - das Time-Driven ActivityBased Costing - dar, das für produktbegleitende Dienstleistungen angepasst wird. Die Umsetzbarkeit und der praktische Nutzen der entwickelten prozessorientierten Planung und Kalkulation werden abschließend in einem Beispiel aus der Unternehmenspraxis dargestellt.

Schlüsselwörter Produktbegleitende Dienstleistungen · Hybride Produkte · Planung · Kalkulation · Time Driven Activity Based Costing

Prof. Dr. K. Möller (

Professur Unternehmensrechnung und Controlling, Georg-August-Universität Göttingen,

Platz der Göttinger Sieben 3, 37073 Göttingen, Deutschland

e-mail: Klaus.Moeller@wiwi.uni-goettingen.de

Dipl. Wirtsch.-Inf. (Univ.) I. Cassack

Mahle SAS Finance France, 175, Route de Colmar, 68040 Ingersheim, France

e-mail: ingo.cassack@fr.mahle.com 


\section{Process Based Planning and Calculation of Product-Accompanying Services}

Summary While the relevance of services increased over the last decades, planning and calculation for services is often disregarded. Instead of following a theoretically clear process services are often offered to the customer unsystematically. In this paper a theoretical based concept for process-oriented planning and calculation of services is developed. The foundation is an adaptation and further improvement of Activity Based Costing. The enhancement with Time-Driven Activity-Based Costing leads to a better adaptation of the concept for product-accompanying services. The realization and practicability of the process-oriented planning and calculation is shown with an example within the mechanical engineering industry.

Keywords Product accompanying services · Hybrid products · Planning · Calculation - Time driven activity based costing

\section{Einführung}

Mit Dienstleistungen werden bereits mehr als 70\% der Bruttowertschöpfung in Deutschland generiert. ${ }^{1}$ Von den Kunden wird zunehmend allerdings nicht nur eine Dienstleistung oder ein physisches Produkt unabhängig voneinander, sondern zunehmend ein Leistungsbündel aus physischem Produkt und Dienstleistung zur Problemlösung erwartet. ${ }^{2}$ Empirische Studien zeigen, dass Wirtschaftlichkeitsaspekte und das Kostenmanagement bei einer derartigen Verbindung eine zentrale Herausforderung darstellen: In rund der Hälfte der Fälle verursachen die Wirtschaftlichkeitsbetrachtung sowie die Planung der Kosten Probleme (vgl. Spath u. Zahn 2003, S. 211 f.). Häufigster Grund dafür ist, dass kernproduktbegleitende Dienstleistungen erst auf Nachfrage durch den Kunden angeboten werden. Ein Defizit ist damit einerseits bzgl. einer systematischen, strategischen Planung und Entwicklung zu konstatieren, andererseits bzgl. des mangelnden Einbezugs von Wirtschaftlichkeitsüberlegungen (vgl. Bullinger u. Scheer 2003, S. 4 ff.; Schwengels 2003, S. 508; Storey u. Kelly 2001, S. 71 ff.).

Wissenschaftliche Publikationen zu Dienstleistungen nehmen zwar seit Mitte der 1980er Jahre kontinuierlich zu, sind jedoch häufig auf den Bereich des Marketings konzentriert. ${ }^{3}$ Dabei steht oft die Qualität von Dienstleistungen im Mittelpunkt, ohne dass auf die Messung und Steuerung der Kosten bzw. Erlöse detail-

\footnotetext{
${ }^{1}$ Vgl. Statistisches Bundesamt (2008). Anfang der 1980er Jahre lag dieser Wert noch bei knapp über 50\%. Zur Bedeutungszunahme vgl. z. B. Corsten (2001, S. 2-19); Meffert u. Bruhn (2003, S. 15).

${ }^{2}$ Vgl. Reiß u. Präuer (2001, S. 48); Spath u. Demuß (2003, S. 468-470); Belz (2000, S. 430 ff). Die Kombination von Sach- und Dienstleistungen wird in der Literatur häufig als hybrides Produkt bezeichnet. Im Weiteren wird die Dienstleistungskomponente des hybriden Produktes als kernproduktbegleitende Dienstleistung bezeichnet; vgl. Kersten et al. (2006, S. 191); Korell u. Ganz (2000, S. 154); Nemeth u. Ohlhausen (2000, S. 173); Reichwald et al. (2000, S. 7) sowie die Ausführungen in Abschn. 2.2.

${ }^{3}$ Vgl. Bieger (2000, S. 303 f.); Meffert u. Bruhn (2003, S. 3 f.); Göbl (2003); Gouthier (2003, S. 12); Stauss u. Bruhn (2003, S. 5); die Gliederung bei Meyer (1998) sowie ebenfalls die große Anzahl der Autoren bei Bruhn u. Meffert (2001), die eine Marketingperspektive vertreten.
} 
liert eingegangen wird. Auch im internationalen Kontext lässt sich diese Beobachtung einer Fokussierung auf Marketingaspekte und die Vernachlässigung von Aspekten des Rechnungswesens fortsetzen. ${ }^{4}$ Die Notwendigkeit für eine umfassendere, theoretische Weiterentwicklung der Ansätze zur Wirtschaftlichkeitsbetrachtung von Dienstleistungen wird entsprechend auch in zahlreichen englischsprachigen Publikationen konstatiert (vgl. Norman 2000, S. 124; Fitzsimmons u. Fitzsommons 2000, S. 485 ff.; Storey u. Kelly 2001, S. 71; Thomke 2003, S. 75). Für ein Controlling von Dienstleistungen existieren erste Ansätze, ${ }^{5}$ die wirtschaftliche Planung von Dienstleistungen ist allerdings noch verhältnismäßig unterentwickelt. Insbesondere die Kostenrechnung und Kalkulation (kern-)produktbegleitender Dienstleistungen werden vielfach als entwicklungsbedürftig charakterisiert (vgl. Weber 2002, S. 1; Engelhardt 2002, S. 49; Meffert 2001, S. 334; Meyer 1998b, S. 1903; Simon 1993, S. 19; Schuh u. Speth 1999, S. 32; Paul u. Reckenfelderbäumer 2001, S. 631; Reckenfelderbäumer 2003, S. 169; Reckenfelderbäumer 2004, S. 209 ff.). Für eine Wirtschaftlichkeitsrechnung ist neben der (unternehmensgetriebenen) Selbstkostenkalkulation auch eine (marktgetriebene) Angebotskalkulation zu entwickeln, um durch eine Verbindung der Markt- und Kostenperspektive eine Ergebnisrechnung erstellen zu können (vgl. Roth 2005; Paul u. Reckenfelderbäumer 2001; Woratscheck 2001). Bei der Angebotskalkulation steht die Prognose von Mehrwert, Zahlungsbereitschaft Preiselastizität etc. aus Kundensicht im Mittelpunkt, was Kernthemen des Marketings sind. Für die Selbstkostenkalkulation ist es wichtig, genaue Kostendaten bezüglich der Erstellung der Leistung im Unternehmen zu erheben, was traditionell Aufgabe der Kostenrechnung und des Controllings ist. Im Folgenden soll eine Konzentration auf die Kostenperspektive erfolgen und ein Konzept zur prozessorientierten Planung und Kalkulation von (kern-)produktbegleitenden Dienstleistungen entwickelt sowie seine praktische Umsetzung dargestellt werden. Dazu wird zuerst das Begriffsverständnis von (kern-)produktbegleitenden Dienstleistungen präzisiert und Besonderheiten des Kostenmanagements von Dienstleistungen dargestellt. Anschließend wird das Konzept in fünf Phasen erläutert. Anhand einer praktischen Umsetzung werden diese fünf Phasen in ihrer konkreten Ausgestaltung vorgestellt. Der Beitrag schließt mit einem Fazit und Ausblick auf zukünftige Forschungen.

\section{2 (Kern-)produktbegleitende Dienstleistungen}

\subsection{Dienstleistungen}

Dienstleistungen ${ }^{6}$ stellen in den Wirtschaftswissenschaften einen vielfältig und kontrovers diskutierten Begriff dar. ${ }^{7}$ In der Dienstleistungsliteratur haben sich vier Ansätze für eine Definition gebildet, die im Folgenden näher erläutert und hinsicht-

\footnotetext{
${ }^{4}$ Vgl. zur Fokussierung auf das Marketing Fitzsimmons u. Fitzsimmons (2000); Hope u. Mühlemann (1997); Norman (2000). Zu Anpassungen durch das Management Accounting vgl. exemplarisch Cooper u. Kaplan (1999, S. 454 ff.); Cooper u. Slagmulder (2003, S. 23 ff.); Pak et al. (2002, S. 84).
} 
lich ihrer Zweckmäßigkeit zur Abgrenzung der Begriffe verwendet werden sollen (Kleinaltenkamp 2001, S. 29 ff.):

- Die enumerative Definition, bei der Dienstleistungen durch Aufzählungen bestimmt werden (z. B. beim Statistischen Bundesamt) (vgl. Meyer 1991, S. 197; Statistisches Bundesamt 2005; Küpper 1991, S. 59; Vikas 1991, S. 234 f.).

- Die Negativdefinition, welche Dienstleistungen als Gegenteil zu physischen Produkten bzw. Sachgütern versteht (vgl. Meffert u. Bruhn 2003, S. 27; Meyer 1991, S. 197; Barth 2003, S. 49).

- Die Bestimmung konstitutiver Merkmale, wobei in tätigkeitsorientierte (vgl. Schüller 1967, S. 19; Pepels 2004, S. 884), potenzialorientierte (vgl. Corsten 2001, S. $21 \mathrm{f}$; Meyer 1991, S. $198 \mathrm{f}$.), prozessorientierte und ergebnisorientierte (vgl. Kleinaltenkamp 2001, S. 33; Corsten 2001, S. 22) Definitionen unterschieden werden kann (vgl. Meffert u. Bruhn 2003, S. 27 ff.; Bruhn 2004, S. 18 f.; Pepels 2004, S. 883 f.).

- Die leistungstypologische Abgrenzung von Dienstleistungen (vgl. Engelhardt et al. 1993; Meffert u. Bruhn 2003, S. 32 ff., S. 404 ff.; Burr 2002, S. 6; Kleinaltenkamp 2001, S. 32 ff.).

Die enumerative Definition und die Negativdefinition sind aus wissenschaftlicher Sicht unbefriedigend. ${ }^{8}$ Im Folgenden sollen auf Basis der übrigen Definitionen einige Charakteristika dargestellt werden, die für das Verständnis von Dienstleistungen zentral erscheinen und zu einem Begriffsverständnis beitragen:

- Potenzialperspektive: Ein Anbieter kann i.d. R. lediglich seine Bereitschaft und sein Potenzial offerieren (z. B. die Fähigkeit zur Durchführung einer komplexen Wartung). Dienstleistungen fungieren daher in einer vorvertraglichen Phase im Sinne eines Leistungsversprechens (vgl. Engelhardt et al. 1993, S. 398 f.; Biermann 1999, S. 22 f.; Meyer 1998a, S. 6 f.; Woratschek 2001a, S. 265; Pepels 2004, S. 884; Frehse 2002, S. 34). Eine Realisierung erfolgt erst durch die Endkombination mit dem externen Faktor. ${ }^{9}$

- Prozessperspektive: Eine Dienstleistung kommt nur durch eine synchrone Interaktion (,Uno-actu-Prinzip“10) zwischen dem Anbieter sowie Nachfrager, d.h. der Gleichzeitigkeit von Produktion und Absatz zustande. Durch die notwendige Beteiligung des ,externen Faktors“, an dem die Zustandsveränderung bewirkt

\footnotetext{
${ }^{5}$ Vgl. Weber u. Schäffer (2001); Corsten u. Gössinger (2003); Borrmann (2003); Fischer (2000); Witt (2003). Zur konkreten Anwendung von Controllinginstrumenten vgl. exemplarisch Niemand (1996); Reckenfelderbäumer (1995); Schuh u. Speth (1999).

${ }^{6} \mathrm{Im}$ Folgenden wird der Begriff der Dienstleistung in Analogie mit der angloamerikanischen Literatur synonym zu „Service“ verwendet. Zu einer analogen Gleichsetzung der beiden Begriffe Dienstleistung und Service vgl. exemplarisch ebenso Meffert u. Bruhn (2003, S. 30); Kleinaltenkamp (2001, S. 29); Spath et al. (2004, S. 281) sowie die dort aufgeführten Erläuterungen.

${ }^{7}$ Vgl. Corsten (2001, S. 21 ff.); Kleinaltenkamp (2001, S. 27 ff.); Meffert u. Bruhn (2003, S. 27 ff.); sowie Rück (2000), der die Geschichte dieses Begriffs in der ökonomischen Theorie ausführlich darstellt. ${ }^{8}$ Vgl. Corsten (2001, S. 21); Kleinaltenkamp (2001, S. 32) und die dort zitierte Literatur.

${ }^{9}$ Ein externer Faktor ist ein vom Kunden in den Dienstleistungsproduktionsprozess eingebrachter Produktionsfaktor (Person, Sache oder immaterielles Gut), vgl. exemplarisch Corsten (2001, S. 22); Engelhardt et al. (1993, S. 401-404).

${ }^{10}$ Zum „Uno-actu-Prinzip“ vgl. Corsten (2001, S. 22) und Bruhn (2004, S. 19).
} 
wird, wird die Leistungsproduktion unmittelbar (ohne Zwischenschaltung anderer Objekte) am Kunden durchgeführt (vgl. Meyer 1998a, S. 6). Der Anbieter von Dienstleistungen hat durch diese Besonderheit weniger Einfluss auf die Realisierung der eigentlichen Produktion als der Anbieter von Sachgütern (vgl. Steven u. Schade 2004, S. 547).

- Ergebnisperspektive: Eine Dienstleistung kann als immaterielles Gut einer Tätigkeit bzw. Verrichtung angesehen werden (vgl. Bruhn 2004, S. 19; Corsten 2001, S. 22). Problematisch werden die klare Herausarbeitung und Bestimmung der immateriellen Eigenschaft von Dienstleistungen, wenn diese in einer engen technischen oder leistungsbezogenen Verbindung mit einer materiellen Sachleistung steht. ${ }^{11}$

Im Rahmen der folgenden Ausführungen wird folgendes Begriffsverständnis zugrunde gelegt: Unter Dienstleistungen werden auf Potenzialen beruhende Leistungsversprechen (Potenzialperspektive) verstanden, deren Erstellung an einem externen Faktor in einem Prozess erfolgt (Prozessperspektive). ${ }^{12}$

Diese beiden notwendigen Eigenschaften charakterisieren Dienstleistungen und lassen sich nie vollständig substituieren. Die Immaterialität (Ergebnisperspektive) wird nicht als konstitutives Merkmal, sondern als Eigenschaftsform verwendet.

\subsection{Verbindung zwischen Sach- und Dienstleistung}

Die Verbindung der Dienstleistung zum physischen Produkt ist das wesentliche, notwendige Merkmal der betrachteten, (kern-)produktbegleitenden Dienstleistungen. ${ }^{13}$ Für die im Fokus stehenden Dienstleistungen muss eine Beziehung zum physischen Produkt bzw. dessen Produktmodell (d.h. die vorläufige Konkretisierung des Produktes z. B. in Form eines Prototyps) vorliegen. Durch diese Einschränkung werden rein immaterielle Dienstleistungen (z. B. reine Finanzierungsoder Vortragsdienstleistungen) aus der vorliegenden Untersuchung ausgeschlossen. Die Verbindung zum physischen Produkt kann sowohl in der Potenzial-, Prozessoder Ergebnisphase der Dienstleistung stattfinden. Der unmittelbare Bezug der hier betrachteten Dienstleistungen zu physischen Produkten stellt eine konstitutive Eigenschaft dar. In der Regel steht das physische Produkt im Mittelpunkt und die Dienstleistung hat (lediglich) ergänzenden Charakter. Um dies zu betonen wird daher von (kern-)produktbegleitenden Dienstleistungen gesprochen.

Durch die Integration des externen Faktors weisen Dienstleistungen häufig einen hohen Individualisierungsgrad auf. Im Vergleich zu physischen Produkten existiert weiterhin (durch die tendenziell höhere Bedeutung der Immaterialität) oft eine geringere Standardisierung bei (kern-)produktbegleitenden Dienstleistungen, die mit einer

\footnotetext{
${ }^{11}$ Vgl. Olemotz (1995, S. 19), der dieses Problem an den anschaulichen Beispielen von Leasing, Miete, Pacht und Reparaturleistungen erläutert.

12 Vgl. Meyer (1998a, S. 6); Meyer (1991, S. 198 f.); und Woratschek (2001a, S. 265), der allerdings ,,individuelle Leistungsversprechen“ zugrunde legt.

${ }^{13}$ Zum Begriff der produktbegleitenden Dienstleistung vgl. u. a. Buttler u. Stegner (1990, S. 934); Killinger (1999, S. 133-135); Kinkel (2003, S. 1); sowie Schneider (1998, S. 10). Pepels (1996, S. 25), verwendet analog den Begriff der produktverbundenen Dienstleistung.
} 
Erschwerung der Planung einhergeht. ${ }^{14}$ Im Rahmen des vorliegenden Ansatzes werden daher zwar die Besonderheiten, die aus der Integration eines externen Faktors resultieren, berücksichtigt. Einmalige, individuelle und damit prozessual nicht planbare (kern-)produktbegleitende Dienstleistungen (z. B. individuelle Beratungen) werden allerdings aus Komplexitätsgründen nicht in den vorliegenden Ansatz integriert. Es erfolgt vielmehr eine Fokussierung auf (teil-)standardisierte (kern-)produktbegleitende Dienstleistungen, die sich durch wiederholende Prozesse auszeichnen.

Einige Autoren fokussieren mit ihrer Definition von (kern-)produktbegleitenden Dienstleistungen lediglich auf spezielle Dienstleistungsarten. So erfolgt z. B. eine Konzentration auf spezielle Nachfrager (nur Unternehmen) oder ein Ausschluss spezieller Anbieter (z. B. keine produzierenden Dienstleister) (vgl. Spath u. Demuß 2003, S. 471 ff.). Im Fokus der weiteren Ausführung stehen demgegenüber (kern-)produktbegleitende Dienstleistungen im weiteren Sinne: Unter (kern-)produktbegleitenden Dienstleistungen werden daher auf Potenzialen beruhende Leistungsversprechen verstanden (Potenzialsicht), die prozessual unter Einbindung eines externen Faktors erstellt werden (Prozesssicht) und sich durch eine Verbindung zum physischen Produkt auszeichnen.

Die (kern-)produktbegleitende Dienstleistung kann sich sowohl auf eigen- als auch fremderstellte Produkte beziehen. Somit können auch verselbstständigte Dienstleistungen (ohne Erstellung der physischen Produkte im gleichen Unternehmen) kernproduktbegleitende Dienstleistungen sein. Durch ein solches Begriffsverständnis werden auch Engineeringdienstleistungen oder technische Wartungen, die an fremden Produkten erfolgen, mit in die Betrachtung integriert.

\section{Prozessorientiertes Kostenmanagement für Dienstleistungen}

\subsection{Kostenrechnerische Spezifika von Dienstleistungen}

Mit den besonderen Merkmalen von (kern-)produktbegleitenden Dienstleistungen gehen wichtige Konsequenzen für die Kostenrechnung und das Kostenmanagement einher. Den größten Kostenblock in Dienstleistungsunternehmen stellen Bereitschaftskosten dar (vgl. Fischer 2000, S. 152). Durch den Aufbau des Leistungspotenzials (Personal, Gebäude, Maschinen und auch Kommunikationstechnik) entstehen Fixkosten, die unabhängig vom Absatz der eigentlichen Leistung anfallen, während die eigentliche Erbringung der Leistung häufig nur geringe Kosten verursacht. Diese Fixkosten haben oftmals Gemeinkostencharakter, da sie sich nur schwer einzelnen Leistungen zuordnen lassen (vgl. Corsten 1990, S. 103; Palloks-Kahlen u. Kuczynski 2000, S. 135; Reckenfelderbäumer 2003, S. 217; Serfling u. Jeiter 1995, S. 321). Die Bereitschaftskosten bestehen dabei zum größten Teil aus Personalkosten, da mit der Integration der Kundenwünsche und zunehmender Immaterialität der Leistung der Anteil an Materialkosten abnimmt (vgl. Reckenfelderbäumer 1995, S. 42). Sie resultieren zum einen aus der Potenzialbereitstellung, da der Anbieter jederzeit

\footnotetext{
${ }^{14}$ Vgl. Paul u. Reckenfelderbäumer (2001, S. 632), die daneben noch auf die unterschiedlichen Qualitätsausprägungen hinweisen.
} 
in der Lage sein muss, die Kundennachfrage zu bedienen, und zum anderen aus der Eigenschaft der Immaterialität, da Dienstleistungen nicht lagerfähig sind und im Moment ihrer Erbringung verbraucht werden (vgl. Reckenfelderbäumer 2003, S. 217).

Aufgrund von Nachfrageschwankungen besteht für Dienstleistungsunternehmen, die ihre Kapazität am potenziellen Spitzenbedarf ausrichten, die Gefahr von Leerkosten durch ungenutzte Kapazitäten. Eine Orientierung am „Normalbedarf“ hingegen birgt das Risiko, zu Spitzenzeiten Kunden nicht bedienen zu können. Eine der wesentlichen Ursachen für die Auslastungsschwankungen stellt der externe Faktor - also die Kunden - dar. Dadurch, dass diese in unterschiedlichem Ausmaße in den Leistungserstellungsprozess eingreifen, erhöht sich die Planungsunsicherheit bezüglich der Kapazitätsbeanspruchung (vgl. Reckenfelderbäumer 1995, S. 45). Die Integrativität und Immaterialität von Dienstleistungen führen dazu, dass viele Dienstleistungen kundenindividuell erbracht werden, was die Bestimmung und Verrechnung auf Kostenträger weiter erschwert. Durch Heranziehen standardisierter Leistungsmodule oder -bausteine in der Kostenrechnung kann dieses Problem jedoch reduziert werden (vgl. Schuh et al. 2000, S. 44; Reckenfelderbäumer 1998, S. 153). Damit wird deutlich, dass die Kostenstrukturen in Dienstleistungsbetrieben mit ihren hohen Gemeinkostenanteilen bei der Verrechnung mit traditionellen Kostenrechnungsinstrumenten zu Problemen führen.

\subsection{Prozesskostenrechnungen für Dienstleistungen}

Als Lösung für die skizzierte Problematik bietet sich die Prozesskostenrechnung an, die ursprünglich für die kostenrechnerische Abbildung der Gemeinkostenbereiche von Produktionsunternehmen entwickelt wurde. ${ }^{15}$ Die Prozesskostenrechnung liefert eine bessere Durchleuchtung und Verrechnung der Gemeinkosten in den unterstützenden Bereichen. Im Gegensatz zur traditionellen Zuschlagskalkulation werden sachlich zusammenhängende Tätigkeiten über die Kostenstellen hinweg zu Prozessen zusammengefasst. Für diese Prozesse werden entsprechende Prozesskostensätze ermittelt. Kritisch festzuhalten ist, dass die Prozesskostenrechnung eine Vollkostenrechnung - konzeptionell mithin einen Rückschritt - darstellt, da sie keine Beschäftigungsschwankungen abbildet. Durch den Zuschlag bzw. die Verteilung von leistungsmengenneutralen Kostenbestandteilen werden derartige Kosten proportional auf die leistungsmengeninduzierten Kosten verteilt, eine wenig verursachungsgerechte Vorgehensweise, die zu weiteren Verfälschungen führt. Entsprechend sind bei der Anwendung der Prozesskostenrechnung limitierende Voraussetzungen zu beachten, um nicht einer verfälschten Kostendarstellung zu unterliegen. Trotz dieser und weiterer konzeptioneller Kritik ${ }^{16}$ ist die Prozesskostenrechnung zu einem Standardverfahren zur prozessualen Abbildung von Dienstleistungsunternehmen geworden, wobei ein unternehmensweiter Einsatz unter der Berücksichtigung der Dienstleis-

\footnotetext{
${ }^{15}$ Vgl. zur Prozesskostenrechnung Horváth u. Mayer (1993, S.16 ff.); Franz (1991, S. 605); Horváth (2006, S. 554); Stoi (1999, S. 25 ff.). Zu einer Weiterentwicklung in einer Kapazitätskostenrechnung vgl. Mayer (1998b). Zum Activity Based Costing (ABC) Cooper u. Kaplan (1999, S. 454), die festhalten, dass ,ABC, from its origins, has been service rather than production-oriented“.

${ }^{16}$ Vgl. dazu bspw. Glaser (1992, S. 276)
} 
tungsspezifika möglich ist. ${ }^{17}$ Im Rahmen der Prozesskostenrechnung kann ein Unternehmen als Prozessmodell mit theoretisch beliebig vielen Ebenen abgebildet werden. In diesem Zusammenhang werden Dienstleistungen als Prozesse aufgefasst.

Das Konzept des Time-Driven Activity-Based Costing (TD ABC) stellt eine Weiterentwicklung von prozessorientierten Kostenrechnungssystemen dar, indem es insbesondere die Thematik der Leerkosten berücksichtigt. ${ }^{18}$ Wie zu Beginn des Kapitels erwähnt sind die Bereitstellungskosten ein wichtiger Bestandteil in Dienstleistungsunternehmen. Dies resultiert aus der Potenzialbereitstellung, um auf schwankende Nachfrage reagieren zu können, sowie aus der Eigenschaft, dass Dienstleistungen nicht lagerfähig sind und im Moment ihrer Erbringung verbraucht werden (vgl. Reckenfelderbäumer 2003, S. 217). Traditionell werden in der Prozesskostenrechnung die Kostensätze durch Division der Prozesskosten durch die erwarteten oder tatsächlichen Kostentreiber-Mengen ermittelt. Sinkt der Auslastungsgrad und werden die Kosten und Kostentreiber-Mengen nicht unmittelbar angepasst (was bei den meisten Kosten für die indirekten Bereiche kaum möglich ist, da es sich um kurz- und mittelfristig fixe Kosten handelt), ergeben sich steigende Prozesskostensätze, die nun auch die Kosten der nicht genutzten Kapazitäten enthalten. Werden diese nun zur Kalkulation herangezogen, entstehen höhere Preise, die zu einem weiteren Absatzrückgang führen (können), was in der Folgeperiode erneut höhere Prozesskostensätze zur Folge hätte (vgl. Kaplan u. Cooper 1999, S. 155 ff.). Um dieses Risiko des „Herauskalkulierens aus dem Absatzmarkt“ auszuschließen, sollte die Ermittlung der Prozesskostensätze auf Basis der Kostentreibermengen erfolgen, die die maximal zur Verfügung stehenden Kapazitäten der Ressourcen berücksichtigen: „When resources can perform multiple activities, capacity must be measured at the resource level, not the activity level“" (Kaplan u. Cooper 1998, S. 292). Dadurch wird ein separater Ausweis von Nutz- und Leerkosten notwendig, was bei der traditionellen Prozesskostenrechnung nicht möglich ist, da die Kostenstellenkapazitäten vollständig auf die Prozesse verteilt und die Prozesskostensätze auf Basis der realisierten Kostentreibermengen berechnet werden.

Eine weitere Problematik bei Dienstleistungen stellt die Variation der Bearbeitungszeiten dar. Die Behandlung mit pauschalen Durchschnittswerten, wie sie in der Prozesskostenrechnung erfolgt, führt entweder zu einer sehr groben Abbildung der Dienstleistung oder zu einer hohen Komplexität des Prozessmodells durch die feine Differenzierung zwischen Dienstleistungs- bzw. Prozessvarianten. Das Konzept des TD ABC vermeidet diese Erhöhung der Komplexität, indem jeder Teilprozess mit einer Zeitverbrauchsfunktion hinterlegt werden kann. Dies ermöglicht, jedem Teilprozess in Abhängigkeit von bekannten Einflussfaktoren, eine verursachungsgerechte, individuelle Sollzeit zuzuordnen (vgl. Coners u. von der Hardt 2004, S. 113). Dadurch erfolgt eine intensitätsmäßige Anpassung an den Komplexitätsgrad des zu

\footnotetext{
${ }^{17}$ Vgl. Paul u. Reckenfelderbäumer (2001, S. 645); zu weiteren Ansätzen zur Kosten- und Erlösplanung von Dienstleistungen Borrmann (2003); Fischer (2000); Niemand (1996); Reckenfelderbäumer (1995); Weber (1999); Witt (2003) oder die praxisorientierten Darstellungen in Kinkel et al. (2003).

${ }^{18}$ Vgl. zu Leerkosten grundsätzlich Schweitzer u. Küpper (2003, S. 376). Zum Time Driven Activity Based Costing vgl. Kaplan u. Anderson (2004, S. 131 ff.); Kaplan u. Anderson (2007); Bruggeman u. Moreels (2003, S. 51 ff.); Coners u. von der Hardt (2004, S. 108 ff.). Zu einem Vergleich zwischen Activity Based Costing und Prozesskostenrechnung Stoi (1999, S. 31-33).
} 
bearbeitenden Objektes. Damit stellt das TD ABC eine Variante der Prozesskostenrechnung dar, die auf die Zeitgetriebenheit von Prozessen abstellt. Sie bietet damit konzeptionell gute Voraussetzungen für die Abbildung von Dienstleistungen und die besondere Berücksichtigung ihrer Spezifika.

\section{Konzeption einer prozessorientierten Kostenplanung und Kalkulation für (kern-)produktbegleitende Dienstleistungen}

Die Ausrichtung einer Planung und Kalkulation von (kern-)produktbegleitenden Dienstleistungen folgt der Systematik der prozessorientierten Kostenrechnung. Verschiedene Arten und Anpassungen von Prozesskostenrechnungen wurden bereits auch für Dienstleistungen im Allgemeinen und spezielle Dienstleistungsbereiche (z. B. der Telekommunikationsunternehmen) durchgeführt. ${ }^{19}$ (Kern-)produktbegleitende Dienstleistungen wurden bisher allerdings noch nicht betrachtet. Im Folgenden soll daher eine Konzeption für die Kostenplanung und Kalkulation von (kern-)produktbegleitenden Dienstleistungen entwickelt werden. Die Konzeption umfasst fünf Schritte, die im Folgenden näher dargestellt werden:

1. Identifikation einer Sachleistung (oder eines Kernprodukts bzw. einer Produktklasse) und (einer) zugehörigen Dienstleistung(en) in einem interdisziplinären Team. Zentral ist hierbei die integrative Betrachtung von Sach- und Dienstleistung gemeinsam von Anfang der Entwicklung an.

2. Genaue Beschreibung der (kern-)produktbegleitenden Dienstleistung mit Hilfe eines Prozessmodells, das die Bezüge zu physischem Produkt und die generellen Arbeitsschritte wiedergibt.

3. Im nächsten Schritt wird das Prozessmodell weiter detailliert, und es erfolgt analytisch eine Tätigkeits- und Teilprozessplanung. Dabei steht die inhaltliche Konkretisierung der notwendigen Prozesse im Vordergrund.

4. Das detaillierte Prozessmodell wird anschließend mit Kapazitäten und Kosten bewertet und anhand bestimmter Kriterien bzw. einer prozessorientierten Verrechnungslogik verdichtet.

5. Abschließend können die aggregierten, prozessorientierten Kosteninformationen für eine Planung genutzt werden. Die Planung ermöglicht einerseits für eine interne Anwendung die Prognose von benötigten Kapazitäten (relevant insbesondere bei internen Kapazitätsengpässen oder der Abschätzung eines neu aufzubauenden Servicebereichs) und andererseits für eine externe Verwendung die Angebotskalkulation für die (kern-)produktbegleitende Dienstleistung. Damit kann auch auf betriebswirtschaftlicher/kostenrechnerischer Ebene eine gemeinsame Betrachtung von physischem Produkt und Dienstleistung erfolgen sowie Synergien und Tradeoffs systematisch berücksichtigt werden.

Die generierten Informationen können für weitere Instrumente des Kostenmanagements eingesetzt werden. Insbesondere die Übernahme in eine marktorientierte

\footnotetext{
${ }^{19}$ Für Anpassungen der Prozesskostenrechnungen an Spezifika von Dienstleistungen vgl. z. B. Fischer (2000, S. 88 ff.); Paul u. Reckenfelderbäumer (2001, S. 627 ff.); Witt (2003, S. 70 ff.); Serfling u. Jeiter (1995, S. 322 ff.).
} 
Zielkostenrechnung (Target Costing) erscheint vielversprechend, um die (unternehmensintern generierten) Kosteninformationen mit einer externen, marktorientierten Sichtweise zu koppeln. Neben der hier vorgestellten Cost-plus-Kalkulation kann so auch eine retrograde Kalkulation erstellt werden, die sicherstellt, dass sowohl das physische Produkt als auch die Dienstleistung allein (ggf. in Kombination) marktfähig sind. Darüber hinaus wird so die Integration des Konzepts in die frühen Phasen der Produktentwicklung stärker institutionalisiert. Im Folgenden sollen die fünf Schritte im Einzelnen dargestellt werden.

\subsection{Identifikation der (kern-)produktbegleitenden Dienstleistung}

Für die Entwicklung von physischen Produkten existieren inzwischen ausgereifte Entwicklungsprozesse und genormte Vorgehenspläne. Für den Bereich der Entwicklung von Dienstleistungen lässt sich dies nicht in ähnlich umfassender Weise konstatieren. Gerade für komplexe Dienstleistungen fehlen noch geeignete Vorgehensweisen und Methoden. Die Dienstleistungsentwicklung kann im Rahmen von systematischen, standardisierten Phasenmodellen unter Verwendung geeigneter Vorgehensweisen, Methoden und Werkzeuge erfolgen, was Gegenstand des Service Engineering ist. ${ }^{20}$ Es umfasst die Phasen von der Ideenfindung bis zur Markteinführung und wird gefolgt vom Service Management, das die Aktivitäten Evaluation und Ablösung beinhaltet. Gerade bei (kern-)produktbegleitenden Dienstleistungen ist es anzustreben, dass die Dienstleistung simultan zum Produkt in einem integrierten Prozess entwickelt wird. Da für die Entwicklung physischer Produkte häufig bereits dezidierte Entwicklungspläne und Meilensteinverfahren bzw. Stage-Gate-Prozesse ${ }^{21}$ fixiert sind, muss sich der Entwicklungsprozess für Dienstleistungen in der Regel in diese Strukturen einfügen. Ziel ist es in dieser Phase, eine (kern-)produktbegleitenden Dienstleistung zu identifizieren, die am Markt verkauft und mit den eigenen Ressourcen generiert werden kann. Dazu werden zwei Teilschritte vorgeschlagen: Ideengewinnung und Ideenbewertung. Am Ende dieser beiden Schritte steht dann die Ideenauswahl:

- Ideengewinnung:

Am Anfang der Ideengewinnung steht die Suche nach Potenzialen für (kern-)produktbegleitende Dienstleistungen. Dabei sind zwei verschiedene Sichtweisen zu unterscheiden:22 Die ,Inside-out-Perspektive“ ist intern fokussiert, indem sie aus vorhandenen Unternehmensressourcen und -kompetenzen mögliche Dienstleistungen ableitet (Was kann das Unternehmen? - Resource-based View). Dazu sollte zuerst eine systematische Erfassung von Unternehmens- bzw. Dienstleistungspotenzialen erfolgen. Methodisch können durch Prozessanalysen entlang der

\footnotetext{
${ }^{20}$ Vgl. zu einem Überblick Bullinger u. Scheer (2003, S. 3 ff.). Im Kontext der Entwicklung und Gestaltung von Dienstleistungen werden dabei auch die Begriffe ,Service Development“ oder ,DienstleistungsInnovation“" verwendet. Vgl. Downar (2003, S. 268).

${ }^{21}$ Im Rahmen einer Meilenstein- oder Stage-Gate-Systematik wird der Innovationsprozess in sequentiell ablaufende Phasen zerlegt. Nach jeder Phase muss das Projekt einen Meilenstein oder ein „Gate“ passieren, an dem über die weitere Durchführung entschieden wird.

${ }^{22}$ Vgl. Luczak u. Hoeck (2004, S. 88); eine Integration der beiden Sichtweisen bietet das „Customer related Service Engineering (CoRSE), vgl. Zahn et al. (2004, S. 216 f.).“
} 
Wertschöpfungskette, ${ }^{23}$ mit Hilfe von Schnittstellenanalysen und Pattern-Search Dienstleistungen erarbeitet werden. Im Zentrum sollte eine Betrachtung der eigenen physischen Produkte stehen und geprüft werden, welche Leistungen dem Kunden bereits jetzt angeboten werden. Häufig ergibt sich hierbei, dass bereits ein erhebliches Spektrum an Leistungen oft unentgeltlich angeboten wird (z. B. Auslegungen, Berechnungen, Inbetriebnahme). Bei der „Outside-in-Perspektive“ orientiert sich das Unternehmen bei der Entwicklung am Kunden sowie am Wettbewerb und stellt somit eine konsequente Marktorientierung sicher (Was will der Kunde? - Market-based View). Bei der Beteiligung des Kunden am Entwicklungsprozess ist eine eher passive Integration (Kunde als Informationsquelle) von einer aktiven Einbindung zu unterscheiden. Methodisch kann hier auf Fokusgruppen sowie Lead-user-Konzepte zurückgegriffen werden (vgl. Schuh et al. 2004, S. 27). Die Suche nach Kundenproblemen unterstützen Suchfeldanalysen oder Kreativitätstechniken. Bei (kern-)produktbegleitenden Dienstleistungen ist diese Vorgehensweise verhältnismäßig einfach, da über die physischen Produkte in der Regel bereits Kundenbeziehungen existieren. Im Rahmen der „,normalen“ Vertriebsaktivitäten können somit gezielt Informationen zu (kern-)produktbegleitenden Dienstleistungen eingeholt werden.

- Ideenbewertung:

Eine Eingrenzung der Dienstleistungsideen im Sinne eines Trichtermodells kann in einem ersten Schritt durch die Anwendung von Checklisten und Portfolios, aber auch anspruchsvollere Methoden wie Nutzwert-Analyse, Conjoint-Analyse oder Quality Function Deployment erreicht werden. Als Ergebnis der Bewertung kann schließlich eine Bewertung und Auswahl einer oder mehrere Dienstleistungen erfolgen.

Neben der Ablauforganisation stellt auch die Aufbauorganisation bei der strukturierten Dienstleistungsentwicklung eine zentrale Herausforderung dar. Während im Bereich der Entwicklung von physischen Produkten die Verantwortlichkeit bei den F\&E-Abteilungen liegt, fehlen bei der Entwicklung von Dienstleistungen oft klar zugeordnete Verantwortlichkeiten (vgl. Bullinger u. Meiren 2001, S. 153). Eine Möglichkeit stellt die Schaffung einer eigenen Organisationseinheit dar, die sich ausschließlich mit der Entwicklung von neuen (kern-produktbegleitenden) Dienstleistungen (d.h. dem Service Engineering) beschäftigt. Die am weitesten verbreitete Form der organisatorischen Einbindung ist die Dienstleistungsentwicklung durch bereits bestehende Organisationseinheiten. Sie bietet den Vorteil der direkten Verfügbarkeit entwicklungsspezifischen Wissens in der jeweiligen Organisationseinheit. Verantwortlich sollten daher Organisationseinheiten sein, die nahe am Kunden angesiedelt sind (z. B. Marketing, Vertrieb, Produktmanagement). Daneben existiert auch die Möglichkeit zur Fremdvergabe von Entwicklungsaufträgen oder der Einkauf bereits komplett entwickelter Dienstleistungen. Problematisch kann dann jedoch die Anpassung des Fremd-Konzeptes an die eigene Unternehmensstruktur werden. Häufig ergibt sich aufbauorganisatorisch ein zeitlich gestaffeltes Vorgehen: Ausgehend von der Schaffung einer (kleinen) eigenständigen Organisationseinheit (die

${ }^{23}$ Vgl. zu einer solchen Analyse speziell für Dienstleistungen Rösner (1998, S. 154 ff.). 
meist beim Produktmanagement angesiedelt ist) entwickelt sich diese im Laufe der Zeit und der zunehmenden Bedeutung der Dienstleistungen zu einer eigenständigen Vertriebseinheit, die dann wiederum institutionell an die Produktentwicklung angeschlossen wird. Damit kann eine hohe Marktorientierung sichergestellt werden, und die Beteiligung weiterer Einheiten erfolgt nach dem Pull-Prinzip, wenn sich inhaltliche Notwendigkeiten ergeben.

Am Ende dieser Phase sind die zu planenden (kern-)produktbegleitenden Dienstleistungen (ggf. in einer Aggregation von mehreren Dienstleistungen) und ihre Beziehungen zu den physischen Produkten identifiziert, beschrieben und bewertet. Im nächsten Schritt kann auf dieser Basis der Untersuchungsbereich im Unternehmen festgelegt werden, für den im Folgenden Prozessanalysen durchzuführen sind. In der Regel ergibt sich durch die Fokussierung auf eine oder einige wenige (kern-)produktbegleitende Dienstleistungen automatisch eine Eingrenzung des Betrachtungsbereichs im Sinne einer Pilotanwendung. Diese erscheint in jedem Fall empfehlenswert, um die Komplexität der folgenden Untersuchungen zu begrenzen. In einem solchen abgegrenzten Teil der Unternehmung können so zunächst Erfahrungen gesammelt werden, die dann zu einer Ausweitung und zu einem Transfer der Prozesskostenplanung auf andere Dienstleistungen führen kann.

\subsection{Entwicklung eines Prozessmodells}

Zur Erstellung eines Gesamtüberblickes über die (kern-)produktbegleitende Dienstleistung und ihre Bezüge zum physischen Produkt soll in dieser Phase ein (grobes) Prozessmodell Anwendung finden. Dazu erfolgt eine Vorstrukturierung der Hauptprozesse durch ein interdisziplinäres Team. Bei diesem Vorgehen kann jede (kern-)produktbegleitende Dienstleistung als ein Hauptprozess dargestellt werden. ${ }^{24}$ So lassen sich beispielsweise die verschiedenen Reinigungsalternativen elektrotechnischer Bauteile (chemische, thermische, mechanische Reinigung und eine Komplettreinigung) als Hauptprozesse interpretieren. Der Entwurf des Modells erfolgt auf Basis von Erfahrungen mit anderen physischen Produkten und Dienstleistungen und in Abstraktion von den konkreten, detaillierten Gegebenheiten des Unternehmens. Ziel ist es, einen Gesamtüberblick über Ablauf, Funktionsweise, Adressaten und Machbarkeit der Dienstleistung zu gewinnen. Dies beinhaltet auch Überlegungen zu notwendigen Gestaltungsmaßnahmen, um neue Dienstleistungen (z. B. eine innovative Schulung) anbieten zu können. Bereits in dieser Phase sollten auch erste Überlegungen zu möglichen Cost Drivers ${ }^{25}$ der Hauptprozesse erfolgen, um die grundsätzliche Bewertbarkeit des Prozessmodells sicherzustellen. Wichtig dabei ist, dass die Mengen der Kosteneinflussfaktoren (z. B. Anzahl an Wartungen) direkt

\footnotetext{
${ }^{24}$ Ein Hauptprozess wird verstanden als ,,eine Kette homogener Aktivitäten, die demselben Kosteneinflußfaktor unterliegen und für die Prozeßkosten ermittelt werden sollen“. Horváth u. Mayer (1993, S. 16). Ein Teilprozess ist hingegen ,,eine Kette homogener Aktivitäten einer Kostenstelle, die einem oder mehreren Hauptprozessen zugeordnet werden kann und für die Prozeßkosten ermittelt werden“. Horváth u. Mayer (1993, S. 17). Analog nehmen auch Cibis u. Niemand (1993, S. 201), sowie Palloks-Kahlen u. Kuczynski (2000, S. 139), eine Gleichsetzung der Dienstleistung mit dem Hauptprozess vor.

${ }^{25}$ Ein Cost Driver ist: „Als Kosteneinflußfaktor die Meßgröße für die Kostenverursachung, aber auch für den Leistungsort eines Hauptprozesses.“ Horváth (2006, S. 553).
} 
quantifizierbar sind. Dies kann besonders dann schwierig werden, wenn der immaterielle Teil der Dienstleistung sehr dominant ist. Am Ende der Vorstrukturierung der Hauptprozesse ist eine Dokumentation der Ergebnisse in einem Prozessmodell sinnvoll, um die Ausgangsbasis für die anschließende Tätigkeits- und Teilprozessermittlung systematisch zu hinterlegen.

Die Trennung zwischen der Vorstrukturierung der Hauptprozesse und der Detaillierung in Teilprozesse mag künstlich und komplex erscheinen, erfüllt aber einen wichtigen Zweck: Die Entwicklung des Prozessmodells beinhaltet wesentlich kreative sowie innovative Elemente und ist mit dem planerischen Ansatz (,Durchdenken der Umsetzung der kernproduktbegleitenden Dienstleistung“) zentral für die strategische Positionierung der Dienstleistung. Entsprechend ist hier auch organisatorisch ein anderer (stärker führungsorientierter) Personenkreis zu integrieren, der unter teilweiser Abstraktion von den Unternehmenszwängen agiert. Insgesamt sollte diese Phase damit stärker eine marktorientierte Vorgehensweise repräsentieren. Die folgende Teilprozessplanung dagegen muss dann die tatsächliche Ressourcensituation widerspiegeln. Entsprechend sind hier Analysen und Interviews auf der Ebene der Prozessbeteiligten und -verantwortlichen zu führen, die auch über detaillierte Kenntnisse zu den physischen Produkten verfügen.

\subsection{Tätigkeits- und Teilprozessplanung}

Die Tätigkeits- und Teilprozessplanung ist die aufwändigste Aufgabe für die Kostenplanung von (kern-)produktbegleitenden Dienstleistungen (vgl. Fischer 2000, S. 92). Sie geht von dem vorher entwickelten (Haupt-)Prozessmodell aus und detailliert dieses weiter in Teilprozesse und Tätigkeiten. Anschließend erfolgt eine Verdichtung dieser Tätigkeiten und Teilprozesse zur (kern-)produktbegleitenden Dienstleistung (kombiniertes Top-down-bottom-up-Vorgehen). Eine solche Methode ermöglicht ebenfalls die Gestaltung von Prozessschritten und die Entscheidung für ein bestimmtes Vorgehen. So kann eine Gestaltung von wertschöpfenden und nicht-wertschöpfenden Prozessen erfolgen, und es lassen sich bisher durchgeführte überflüssige Teilprozesse eliminieren. ${ }^{26}$

Neben der Unterscheidung in lmi- und lmn-Prozesse ${ }^{27}$ erfolgt eine zusätzliche Untergliederung der Prozesse hinsichtlich ihres Bezugs zum physischen Produkt. So werden die Dienstleistungsteilprozesse in Prozesse ersten, zweiten und dritten Grades differenziert (vgl. Abb. 1). ${ }^{28}$ Prozesse ersten Grades weisen dabei eine direkte Verbindung zum physischen Produkt auf (z. B. eine chemische oder thermische Reinigung). Eine Veränderung am physischen Produkt hat so unmittelbare Auswirkungen auf die Potenziale, den Prozess und das Ergebnis der (kern-)produktbegleitenden Dienstleistungen. Ein indirekter Bezug zum physischen Produkt lässt sich bei Prozessen zweiten Grades herstellen (z. B. Entwicklungs- oder Marketingleistungen). Bei

\footnotetext{
${ }^{26}$ Vgl. zu einer solchen Prozessbereinigung Niemand (1996, S. 89-93).

${ }^{27}$ Vgl. zu dieser generellen Differenzierung von leistungsmengeninduzierten (lmi) und leistungsmengenneutralen (lmn) Kosten im Rahmen der Prozesskostenrechnung z. B. Horváth u. Mayer (1989, S. 216).

${ }^{28}$ Zur Unterscheidung in Prozesse ersten bis dritten Grades vgl. auch Reckenfelderbäumer (1995, S. 122 ff.); Palloks-Kahlen u. Kuczynski (2000, S. 139 ff.).
} 


\begin{tabular}{|l|l|l|}
\hline \multicolumn{1}{|c|}{$\begin{array}{c}\text { Verbindung zum } \\
\text { physischen Produkt }\end{array}$} & \multicolumn{1}{|c|}{ Beispiele } & $\begin{array}{l}\text { Unterstützung der Planung } \\
\text { anhand des Produktes }\end{array}$ \\
\hline $\begin{array}{l}\text { Prozesse 1. Grades } \\
\text { (direkte Verbindung zum } \\
\text { physischen Produkt) }\end{array}$ & $\begin{array}{l}\text { - Chemische Reinigung } \\
\text { - Thermische Reinigung } \\
\text { - Mechanische Reinigung }\end{array}$ & $\begin{array}{l}\text { - Direkte Analyse der Ablaufvarianten } \\
\text { - Entscheidung über mögliche } \\
\text { Prozesstreiber } \\
\text { - Dokumentation der Ergebnisse für } \\
\text { eine iterative Verbesserung }\end{array}$ \\
\hline $\begin{array}{l}\text { Prozesse 2. Grades } \\
\text { (indirekte Verbindung zum } \\
\text { physischen Produkt) }\end{array}$ & - Entwicklungsleistung & $\begin{array}{l}\text { - Indirekte Analyse notwendiger } \\
\text { allgemeiner Entwicklungsleistungen } \\
\text { - Entscheidung über Marketing } \\
\text { - Dokumentation der Ergebnisse und } \\
\text { evtl. iterative Verbesserung }\end{array}$ \\
\hline $\begin{array}{l}\text { Prozesse 3. Grades } \\
\text { (keine oder sehr lose Verbindung } \\
\text { zum physischen Produkt) }\end{array}$ & - Geschäftsführung & $\begin{array}{l}\text { - Unterstützung durch das Produkt } \\
\text { nicht vorteilhaft }\end{array}$ \\
\hline
\end{tabular}

Abb. 1 Prozessdifferenzierung in Abhängigkeit von der Verbindung zum physischen Produkt

diesen Dienstleistungen resultiert nur eine mittelbare Reaktion auf die Veränderung des physischen Produktes. Prozesse dritten Grades umfassen schließlich Aktivitäten, die keine oder nur eine sehr lose Verbindung zum physischen Produkt haben (z. B. Geschäftsbereich leiten). Die Bestimmung der Teilprozesse und die Ermittlung der Teilprozesskosten haben wesentliche Auswirkungen auf die Aussagekraft der Kalkulation. Durch die Differenzierung der Prozesse vom ersten bis zum dritten Grad in Abhängigkeit vom physischen Produkt kann der Produktbezug direkt in der Rechnung mitverwendet werden. Dadurch lassen sich dann auch Schwankungsbreiten und damit Genauigkeitsstufen in der Kalkulation identifizieren.

Generell können die für die Tätigkeits- und Teilprozessplanung notwendigen Informationen durch schriftliche Fragebögen oder persönliche Befragung erhoben werden. ${ }^{29}$ Vorteile für eine schriftliche Befragung liegen in der besseren Vergleichbarkeit der Ergebnisse sowie der Möglichkeit für Mitarbeiter, die Fragen besser durchdenken zu können; problematisch sind die geringe Flexibilität und das Fehlen von persönlichen Erläuterungen. Daher erscheint eine persönliche, mündliche Befragung sinnvoller, da sie in der Lage ist, die spezielle Situation im Unternehmen abzubilden. ${ }^{30}$

Methodisch bietet sich für diesen Arbeitsschritt die Methode des Service Blueprinting an (vgl. Shostak 1984, S. 133 f.). Ein Service Blueprint kann als eine Kombination von Organisationsschaubild und Flussdiagramm beschrieben werden, bei der der Dienstleistungsentstehungsprozess, die beteiligten Bereiche und Kostenstellen sowie die Kontakte des Kunden mit der Dienstleistung in chronologischer

\footnotetext{
${ }^{29}$ Vgl. Fischer (2000, S. 92). Auch die dort vorgeschlagene Auswertung jüngerer Gemeinkostenanalysen kann bedingt als Ausgangspunkt für die Tätigkeits- und Teilprozessplanung eingesetzt werden.

${ }^{30}$ Allerdings sei an dieser Stelle ebenfalls auf den großen Aufwand bei diesem Vorgehen hingewiesen. So stellen Kaplan u. Anderson (2003, S. 1) in Analogie für das ABC fest: „The traditional ABC model has been difficult for many organizations to implement because of the high costs incurred to interview and survey people“.
} 
Abfolge transparent aufgezeigt werden. Kontaktprozesse laufen dabei im so genannten „Front Office“-Bereich ab, autonome Prozesse, die der Kunde meist nicht wahrnimmt, im „Back Office“-Bereich. Die Prozessarten werden im Schaubild getrennt durch die „Line of Visibility“. Diese Methode eignet sich sowohl zur Planung neuer Dienstleistungen als auch zur Analyse und Optimierung bestehender Dienstleistungsangebote, da z. B. redundante Prozesse oder mögliche Fehlerquellen aufgezeigt werden können.

\subsection{Kapazitäts- und Kostenplanung}

Die Planung der für die (kern-)produktbegleitenden Dienstleistungen notwendigen Zeiten und Kapazitäten (und die sich daraus ergebenden Kosten) ist grundsätzlich mit zwei Vorgehensweisen möglich (vgl. Mayer 1998, S. 146 f.; Witt 2003, S. 78 f.):

- Bei einem retrograden Top-down-Ansatz wird zunächst die gesamte Mitarbeiterkapazität geplant und anschließend auf die betroffenen Teilprozesse verteilt. Aus der Division von Kapazitäten in Mitarbeiterjahren (MJ) durch die Maßgrößenmengen ergeben sich die Kapazitätsanteile je einmaliger Durchführung eines Teilprozesses. Das Verfahren erscheint insbesondere dann anwendbar, wenn die Teilprozesszeiten sehr stark schwanken und schwer prognostizierbar sind. Weiterhin ist es aufgrund der aggregierten Darstellung weniger aufwändig und schneller durchführbar, als das Bottom-up-Vorgehen. Allerdings wird mit diesem Verfahren keine Detailplanung und Detailanalyse für die (kern-)produktbegleitenden Dienstleistungen unterstützt, und insbesondere die Einbindung in ein TD ABC erscheint schwer möglich. ${ }^{31}$

- Ausgangspunkt für ein Bottom-up-Vorgehen bei der Planung von Kapazitäten und Zeiten ist die Dauer (in Minuten) für die Durchführung einzelner Teilprozesse der (kern-)produktbegleitenden Dienstleistungen. Die Menge der geplanten Teilprozesse multipliziert mit den Minutenwerten für die Durchführung ergibt die Jahresarbeitsminuten für die betrachtete Kostenstelle. ${ }^{32}$ Damit ergibt sich die Sollarbeitszeit pro Mitarbeiter und Jahr ohne Rüst-, Verteil- und Leerzeiten.

Das Bottom-up-Vorgehen liegt als primäres Verfahren dem vorliegenden Konzept zugrunde. So können die Informationen aus der Tätigkeitsanalyse und Teilprozessplanung direkt umgesetzt und damit Zeiten und Kapazitäten bestimmt werden. Als problematisch ist allerdings festzuhalten, dass eine Rückkopplung mit den im Unternehmen vorhandenen Ressourcen nur indirekt gegeben ist. So ist gerade bei innovativen (kern-)produktbegleitenden Dienstleistungen zu klären, ob für die geplante Durchführung von Teilprozessen auch angemessene Kapazitäten (u. a. hin-

\footnotetext{
${ }^{31}$ Für die Planung fehlen hierdurch häufig Sollzeiten, und eine Abweichungsanalyse ist nicht bzw. nur sehr begrenzt möglich. Vgl. Bruggeman u. Moreels (2003, S. 62 ff.); Coners u. von der Hardt (2004, S. $110 \mathrm{ff}$.).

${ }^{32}$ Die geplanten Zeiten sind dabei hinsichtlich ihrer Adäquanz zu hinterfragen und gegebenenfalls mit anderen Zeiten, z. B. aus REFA-Studien, abzugleichen. Vgl. Serfling u. Jeiter (1995, S. 323 f.).
} 
reichend qualifizierte Mitarbeiter) vorhanden sind. ${ }^{33}$ Dafür können auch Top-downInformationen z. B. von Kostenstellenkosten mit in die Konzeption eingehen.

Zur Vereinfachung der Planung bzw. Rechnung werden im vorliegenden Konzept in Anlehnung an das TD ABC zwei Parameter geschätzt: ${ }^{34}$

- Zum Ersten ist die Betrachtung der Stundenkostensätze für die betrachtete Kostenstelle erforderlich. ${ }^{35}$ Diese ergeben sich durch eine Division der geplanten Kostenstellenkosten durch die geplante Nettokapazität der Kostenstelle. Die Nettokapazität errechnet sich dabei aus der Gesamtarbeitszeit der Mitarbeiter der Kostenstelle (in Stunden) abzüglich einer geschätzten, nicht-produktiven Zeit für Krankheit, Urlaub und Verteilzeiten (vgl. Coners u. von der Hardt 2004, S. 111). Kaplan und Cooper setzen für diese Nettokapazität (,Practical Capacity“) einen Wert von 80 bis $85 \%$ der Gesamtarbeitszeit an (vgl. Kaplan u. Cooper 1998, S. 127).

- Zum Zweiten erfordert das TD ABC die Schätzung der Sollzeiten, die zur einmaligen Prozessdurchführung erforderlich sind. Für diese Zeiten ist keine absolute Genauigkeit erforderlich, vielmehr sind sie im Sinne einer Tendenzaussage zu verstehen. ${ }^{36}$ Die Annahmen dieser Sollzeiten ersetzen dabei die aufwändigen erhobenen Vermutungen zur prozentualen Verteilung von Gesamtarbeitszeiten auf einzelne Aktivitäten.

Beim Bottom-up-Vorgehen zur Kostenplanung sollte auch der Grad der Abhängigkeit der Teilprozesse vom physischen Produkt für mögliche Planungszyklen bzw. Iterationsschleifen berücksichtigt werden, da diese Auswirkungen auf die Genauigkeit und Schwankungsbreite der Zeiten sowie Kosten hat. Für Prozesse ersten Grades und bedingt auch für die Prozesse zweiten Grades kann durch eine weitere Planungsund Abstimmungsrunde die Genauigkeit oftmals erheblich verbessert werden. Für Teilprozesse dritten Grades, die keine oder nur eine sehr lose Beziehung zum physischen Produkt aufweisen, ist aus pragmatischen Gesichtspunkten in der vorliegenden Konzeption keine weitere Iterationsschleife vorgesehen, da durch diese nur sehr eingeschränkt mehr Informationen generiert werden können.

\subsection{Planung und Kalkulation}

Im abschließenden Schritt kann eine Kostenplanung bzw. Kalkulation der kostenstellenübergreifenden Hauptprozesse - also der (kern-)produktbegleitenden Dienstleis-

\footnotetext{
${ }^{33}$ Zur Berücksichtigung von angemessenen Kapazitäten und Kompetenzen gerade bei der Neuentwicklung von Dienstleistungen vgl. Stanik (2004, S. 20 f.).

${ }^{34}$ Vgl. Kaplan u. Anderson (2004, S. 132 ff.); Kaplan u. Anderson (2003, S. 6 ff.), sowie für eine Anwendung Coners u. von der Hardt (2004, S. 110 ff.).

${ }^{35}$ Kaplan und Anderson benennen dies ,unit cost“ und berechnen die Kosten pro Minute für die Durchführung einer Aktivität. Vgl. Kaplan u. Anderson (2003, S. 7). In Anlehnung an die Gegebenheiten im deutschen Rechnungswesen werden diese ,unit cost“ nicht als Stückkosten, sondern als ein Stundenkostensatz aufgefasst. Diese Stundenbetrachtung geschieht auch aus Gründen einer besseren Vergleichbarkeit. Eine Umwandlung in Minuten ist abhängig von den betrachteten Teilprozessen, jedoch auch möglich.

${ }^{36}$ Kaplan u. Anderson (2003, S. 7), stellen dazu fest: „Precision is not critical; rough accuracy is sufficient".
} 
tungen - erfolgen. Bei der Zurechnung können im Rahmen einer Analyse verschiedene Alternativen der Verdichtung bewertet, eine Entscheidung über die Zurechnung vorgenommen und anschließend für eine mögliche Iterationsschleife dokumentiert werden. Diese Zurechnungen von Kosten für die (kern-)produktbegleitenden Dienstleistungen können dabei auch anteilig erfolgen, indem für den Hauptprozess nur die Kosten geplant und kalkuliert werden, die diesem direkt zugeordnet werden können. ${ }^{37}$ Als Ergebnis dieses Vorgehens stehen für die (kern-)produktbegleitenden Dienstleistungen prognostizierte Prozessstandardkosten zur Verfügung. Auf dieser Basis können im Zuge einer Cost-Plus-Kalkulation nun die Kosten für das physische Produkt und die begleitende Dienstleistung unabhängig berechnet werden. In einer Ergebnisrechnung können dann unter Berücksichtigung von Absatz- und Preisprognosen Geschäftsmodelle für die Produktbündel entwickelt werden. Entsprechend den Prognosen können hier verschiedene Szenarien analysiert werden, die von einer unabhängigen Vermarktung von physischem Produkt und Dienstleistung über verschiedene Integrationsstufen bis $\mathrm{zu}$ einem integrativen Angebot aus Produkt und begleitender Dienstleistung reichen. Für jede Variante kann auf Basis einer Marktabschätzung und der mit der o. a. Systematik berechneten Kosten für die Dienstleistung in Verbindung mit den Kosten für das physische Produkt eine Wirtschaftlichkeitsanalyse (Absatz-Sensitivitäten, Portfolio-Betrachtung für die Positionierung etc.) erfolgen. Daraus abgeleitet kann dann systematisch eine Preis-Absatz-Strategie für Produkt und (kern-)produktbgeleitende Dienstleistung festgelegt werden - mithin das Ziel einer systematischen Einbeziehung von (kern-)produktbegleitenden Dienstleistungen in Entwicklung und Vermarktung realisiert werden.

\section{Beispiel der prozessorientierten Kostenplanung einer Wartungs-Dienstleistung}

Im Folgenden wird die Umsetzung der Kostenplanung und Kalkulation für (kern-) roduktbegleitende Dienstleistungen anhand der technischen Wartung bei einem Werkzeughersteller näher beschrieben. Die Geräte $\mathrm{GmbH}^{38}$ fertigt Präzisionswerkzeuge und erwirtschaftet mit ca. 750 Mitarbeitern einen Umsatz von 70 Millionen Euro. Die Produkte werden in zwei Geschäftsfelder unterschieden: Werkzeug- und Werkstückhalter sowie elektromechanische Maschinenkomponenten. Sie werden primär im Werkzeug- und Maschinenbau, aber auch in zahlreichen weiteren Branchen (z. B. der Verpackungsindustrie) eingesetzt. Das angebotene Produktspektrum umfasst insgesamt fast 10.000 Varianten. Die Tooling $\mathrm{GmbH}$ zeichnet sich durch eine hohe internationale Orientierung aus und besitzt zahlreiche ausländische Vertriebsgesellschaften und Vertretungen. Das Angebot hat sich von kostenbetonten Standardprodukten hin zu innovativen High-End-Produkten verändert. Kernproduktbegleitende Dienstleistungen (z. B. Wartungen, Engineering, Schulungen) werden auf

\footnotetext{
${ }^{37}$ Vgl. Niemand (1996, S. 102). Mayer (1998, S. 149) schlägt auch für eine Zurechnung und Verdichtung die Trennung in lmi- und lmn-Kosten vor, damit in Abhängigkeit von der Entscheidungssituation die jeweils relevanten Kosten zur Verfügung stehen.

38 Aufgrund der Vertraulichkeit wird das Unternehmen anonymisiert dargestellt.
} 
Kundenwunsch in beiden Geschäftsfeldern angeboten. Ausgehend von den physischen Produkten wird so angestrebt, einen Leistungskranz an ergänzenden Dienstleistungen für die Kunden anzubieten.

\subsection{Identifikation der (kern-)produktbegleitenden Dienstleistung}

Wartungen haben für Werkzeughalter eine wesentliche Bedeutung. Da durch die kontinuierliche Weiterentwicklung der Maschinen immer höhere Maximalbelastungen (z. B. durch sehr hohe Drehzahlen) erfolgen, unterstützt eine proaktive Wartung eine lange Standzeit und geringe Ausfallzeiten. Die Kunden fragen daher - in Ergänzung zu den physischen Werkzeughaltern - verstärkt lebenszyklusbezogene bzw. langfristige Wartungsverträge nach. Für die Geräte GmbH ergibt sich hier das Problem, derartige Dienstleistungen verursachungsgerecht zu bepreisen und zu kalkulieren. Da bei Wartungen für die Zukunft ein stark wachsendes Absatzpotenzial gesehen wird und sie sich durch standardisierte Tätigkeiten sowie repetitive Teilprozesse auszeichnen, wurden sie für die Anwendung des Konzeptes ausgewählt. Im Fallbeispiel war daher eine aufwändige Identifikation (kern-)produktbegleitender Dienstleistungen weder sinnvoll noch notwendig, da die Markt- bzw. Kundenanforderungen eindeutig waren. Diese Situation ist typisch für Branchen wie den Maschinenbau, in denen die reine Existenz von (kernproduktbegleitenden) Dienstleistungen häufig bereits ein Differenzierungsmerkmal darstellt. In gesättigten Märkten dürfte der Aufwand zur Identifikation von potenziell erfolgreichen Dienstleistungen mit den in Abschn. 4.1. beschriebenen Methoden erheblich höher liegen. Ausgehend von der grundsätzlichen Dienstleistung ,,technische Wartung“ erfolgte eine weitere Fokussierung für die pilothafte Umsetzung der Konzeption. Die erste Konzentration erfolgte hinsichtlich des ausgewählten Produktes auf Spannfutter. Ausgehend vom bisherigen Ablauf von Wartungen konnten so die Kostenstellen „Kundendienst“ sowie ,Vertrieb Spannfutter" als wesentliche Unternehmensteile identifiziert werden. Darüber hinaus erfolgte eine weitere Einengung der Betrachtung auf drei verschiedene Spannfutterklassen.

\subsection{Prozessmodell}

Die Vorstrukturierung der Hauptprozesse bzw. die Erstellung eines ersten Prozessmodells erfolgte auf Basis des bestehenden Produktportfolios in Diskussion mit der ersten Führungsebene aus den Bereichen Entwicklung, Controlling und Vertrieb. Neben der Berücksichtigung von bestehenden Dienstleistungen wurden ebenfalls zukünftige Dienstleistungen und damit verbundene strategische Möglichkeiten diskutiert und analysiert. Parallel dazu erfolgt eine Analyse der Cost Driver. Das Prozessmodell für die (kern-)produktbegleitenden Dienstleistungen umfasste 28 Hauptprozesse. Zur Illustration erfolgt beim weiteren Vorgehen eine Konzentration auf den Hauptprozess ,Wartung“, weil dieser aus Kundensicht hohe Bedeutung hat. Aus der Diskussion über zukünftige Dienstleistungsoptionen wurde bereits ersichtlich, dass die Möglichkeit besteht, verschiedene Hauptprozesse zu einem Full-Service-Paket zu koppeln. Ein solcher Ausbau von Full-Service-Paketen wird für die Zukunft bei der Geräte $\mathrm{GmbH}$ angestrebt. 


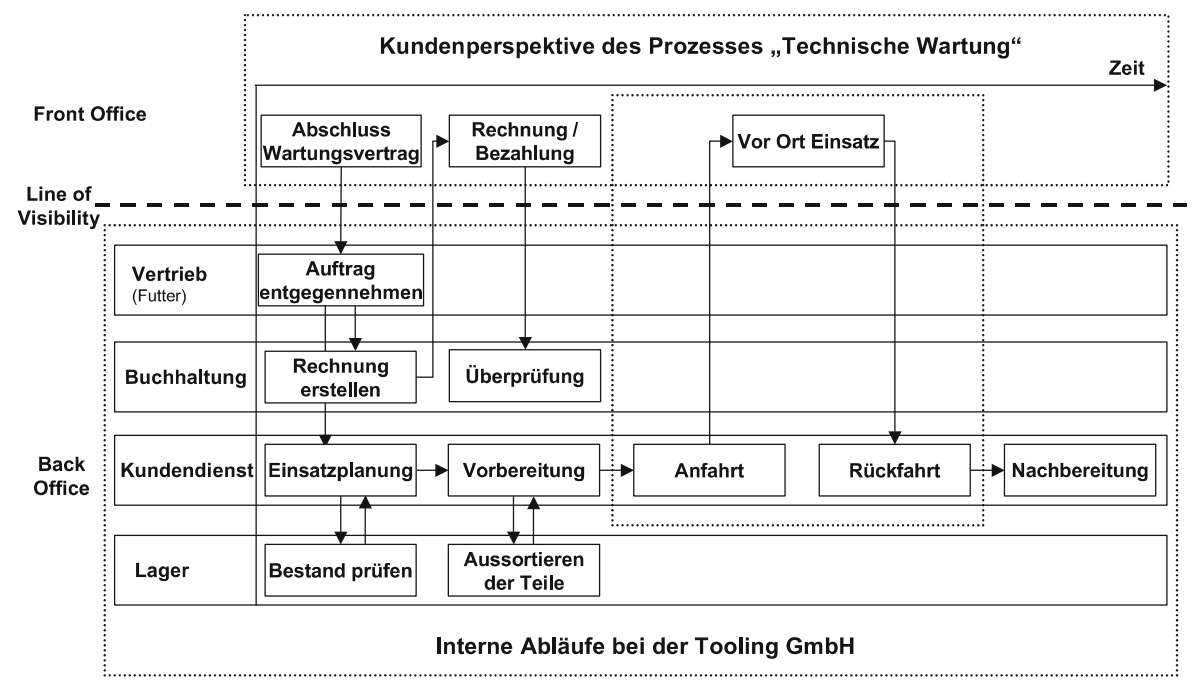

Abb. 2 Service Blueprint für die technische Wartung

\subsection{Tätigkeits- und Teilprozessplanung}

Ein genereller Überblick über die bei einer technischen Wartung ablaufenden Teilprozesse wurde zunächst mit einem Service Blueprint dargestellt (vgl. Abb. 2). Auch ließ sich damit die Mitwirkung der beteiligten Backoffice-Bereiche und Abteilungen aufzeigen und Schnittstellen zwischen den Bereichen identifizieren.

\subsection{Kapazitäts- und Kostenplanung}

Die Kapazitäts- und Kostenplanung für die Geräte $\mathrm{GmbH}$ erfolgte primär mit dem Bottom-up-Verfahren. Wichtig war bei der Bottom-up-Planung, dass die gesamten Kapazitäten der Kostenstelle auf die einzelnen Teilprozesse umgelegt wurden. Beispielhaft soll dies an der Kostenstelle „Kundendienst“ aufgezeigt werden (vgl. Abb. 3). Die erste Schätzung der Dauer von Teilprozessen einer technischen Wartung konnte auf Basis ähnlicher, bisher existierender Produkte abgeleitet werden. So konnte die angewandte Technik (Kraft- oder Handspannfutter), die Anzahl der Verschleißteile und die geplante Produktgröße identifiziert werden. Dabei wurden drei zukünftige Produktgrößenklassen differenziert, bei denen die Zeit der Wartung für die Prozesse ersten Grades mit der Größe steigt. (In der Rechnung wurden die drei Produktklassen daher mit drei Äquivalenzziffern für die unterschiedlichen Teilprozesse abgebildet. ${ }^{39}$ In einem weiteren Planungszyklus erfolgten anschließend eine Überprüfung und zum Teil eine Veränderung der Sollzeiten. Ausgehend von der geplanten Kapazität für die Kostenstelle wurde die Nettokapazität (ohne Zeiten für Urlaub, Krank-

\footnotetext{
${ }^{39}$ Vgl. zu einer ähnlichen Vorgehensweise mit Äquivalenzziffern bzw. Komplexitätsindizes Kaplan u. Cooper (1998, S. 98f.).
} 


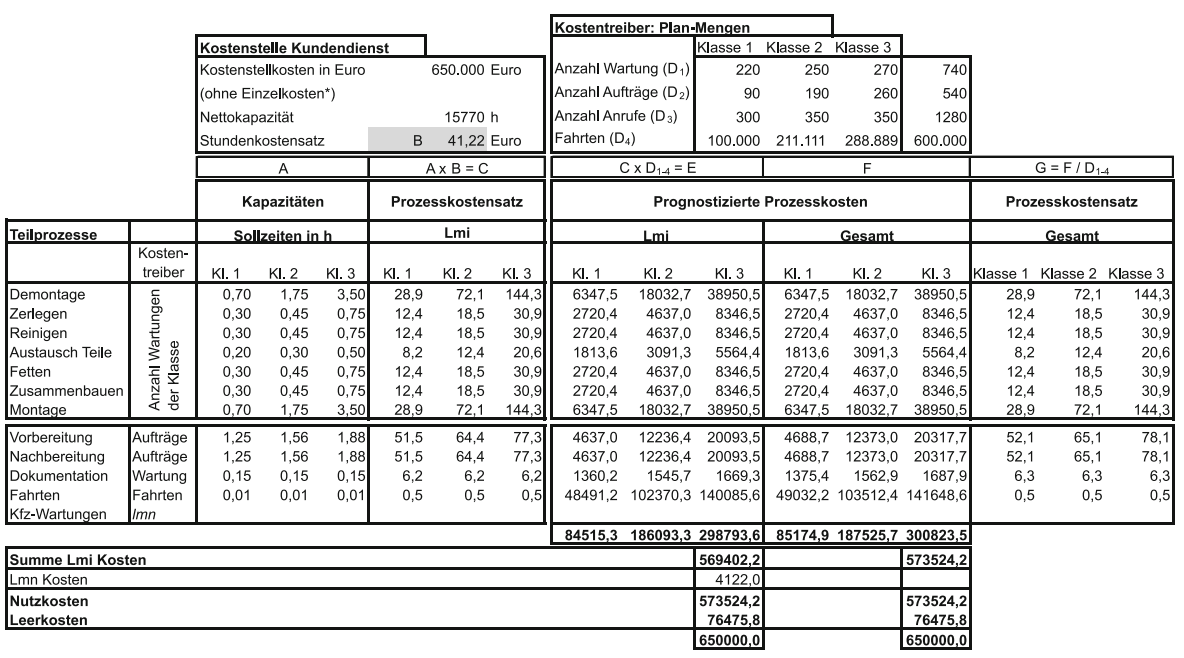

Abb. 3 Prozesskosten und Prozesskostensätze für die Kostenstelle „Kundendienst“

heit und ohne Verteilzeiten) von 15.770 Stunden ermittelt. Die gesamten Kostenstellenkosten umfassten auch Strom- und Raumkosten sowie Abschreibungen und sonstige Gemeinkosten. Insgesamt betrugen die Kostenstellenkosten 650.000€, wobei die Personalkosten mit ca. 92\% dominierten. Aus der Division der Kostenstellenkosten durch die Nettokapazität resultierte der Stundenkostensatz von $41,22 €$.

Der lmi-Prozesskostensatz für die einzelnen Teilprozesse resultiert aus der Multiplikation der ermittelten Sollzeiten (A) mit dem Stundenkostensatz (B). Für den Teilprozess „Demontage“ eines Futters Klasse 1 resultieren so $0,7 \mathrm{~h} \times 41,22 € / \mathrm{h}=$ 28,85€. Multipliziert man dieses Produkt (C) mit den aus der Absatzplanung prognostizierten Mengen der Kostentreiber $\left(\mathrm{D}_{1-4}\right)$, so ergeben sich die lmi-Prozesskosten. Bei der Planung der Demontage waren dies 6347,5€.40 Nach einer Umlage der lmn-Kosten (F) und anschließender Division durch die Kostentreiber resultiert der geplante Gesamtprozesskostensatz (G).

Die iterative Planung in mehreren (hier: zwei) Runden zeigte, dass Schwankungsbreiten reduziert werden konnten, da bei einem zweiten Planungslauf in der Regel mehr und bessere Informationen vorliegen. Ein solches Vorgehen reduziert also Unsicherheit, produziert aber auch einen höheren Aufwand. Über die Anwendung und Intensität derartiger Zyklen ist daher im Einzelfall und unter Berücksichtigung von Kosten-Nutzen-Erwägungen zu entscheiden.

\subsection{Kalkulation}

Die Dienstleistung ,technische Wartung“ wurde im vorliegenden Fall als Hauptprozess angesehen. Auf Basis der Hauptprozessverdichtung konnte so die Plan-

\footnotetext{
${ }^{40}$ Der Wert resultiert aus der Mulitplikation des Prozesskostensatzes „Demontage“ (28,854€) mit 220 geplanten Wartungen.
} 


\begin{tabular}{|l|c|r|}
\hline Kalkulationsmengen & \multicolumn{1}{|c|}{} \\
\hline \multicolumn{1}{|l|}{ Klasse 1 Klasse 2 } & Klasse 3 \\
\hline Anzahl Wartungen & 10 & 10 \\
Anzahl Aufträge & & 1 \\
Anzahl Anrufe & & 10 \\
km & & 200 \\
Laufzeit in Jahren & & 3 \\
\hline
\end{tabular}

\begin{tabular}{|c|c|c|c|c|c|c|c|}
\hline & & & \multicolumn{3}{|c|}{ Einmalige Durchführung } & \multicolumn{2}{|c|}{ Auftrag } \\
\hline & & & Medium & Min & Max & Min & Max \\
\hline $\begin{array}{l}\text { Produkt- } \\
\text { bezug }\end{array}$ & Teilprozesskosten & Kostentreiber & & & & & \\
\hline \multirow{7}{*}{ 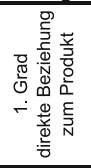 } & Demontage & Wartung & 288,5 & 250,9 & 326,2 & 752,7 & 978,5 \\
\hline & Zerlegen & Wartung & 123,7 & 89,9 & 157,4 & 269,8 & 472,1 \\
\hline & Reinigen & Wartung & 123,7 & 98,9 & 148,4 & 296,8 & 445,1 \\
\hline & Austausch Verschleißteile & Wartung & 82,4 & 60,0 & 104,9 & 179,9 & 314,8 \\
\hline & Fetten & Wartung & 123,7 & 98,9 & 148,4 & 296,8 & 445,1 \\
\hline & Zusammenbauen & Wartung & 123,7 & 89,9 & 157,4 & 269,8 & 472,1 \\
\hline & Montage & Wartung & 288,5 & 250,9 & 326,2 & 752,7 & 978,5 \\
\hline \multirow{16}{*}{ 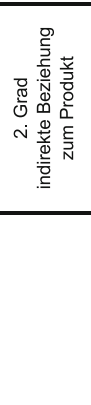 } & Kundenkontakt & Anrufe & 134,9 & 134,9 & 134,9 & 404,8 & 404,6 \\
\hline & Vertragsabschluss & Aufträge & 27,0 & 27,0 & 27,0 & 27,0 & 27,0 \\
\hline & Auftragsabwicklung & Aufträge & 54,0 & 54,0 & 54,0 & 54,0 & 54,0 \\
\hline & Aussortieren Teile & Wartung & 184,9 & 158,8 & 215,4 & 476,3 & 646,2 \\
\hline & Vorbereitung & Aufträge & 52,1 & 49,5 & 54,6 & 148,5 & 163,7 \\
\hline & Nachbereitung & Aufträge & 52,1 & 49,5 & 54,6 & 148,5 & 163,7 \\
\hline & Dokumentation & Wartung & 62,9 & 62,9 & 62,9 & 188,8 & 188,8 \\
\hline & Fakturierung & Aufträge & 46,3 & 46,3 & 46,3 & 46,3 & 46,3 \\
\hline & Fahrten & Entfernung & 98,7 & 98,7 & 98,7 & 296,1 & 296,1 \\
\hline & \multirow{2}{*}{\multicolumn{2}{|c|}{$\begin{array}{l}\text { Hauptprozesskosten } \\
\text { Prozesskosten Wartungsvertrag }\end{array}$}} & $1.867,0$ & $1.621,0$ & $2.116,9$ & & \\
\hline & & & & & & $4.608,5$ & $6.096,4$ \\
\hline & \multicolumn{2}{|l|}{ Einzelkosten } & & & & & \\
\hline & Material & & & 550,0 & 550,0 & $1.650,0$ & $1.650,0$ \\
\hline & Fahrtkosten & & & 70,0 & 70,0 & 210,0 & 210,0 \\
\hline & Prozesse 3. Grades & (GK-Umlage) & & & & 417,7 & 417,7 \\
\hline & \multicolumn{2}{|l|}{ Kosten des Auftrages } & & & & $6.886,2$ & $8.374,2$ \\
\hline
\end{tabular}

Abb. 4 Hauptprozessverdichtung und Kosten eines Standardauftrags

Kalkulation eines mehrjährigen Wartungsauftrages erfolgen, bei dem einzelne Teilprozesse mehrfach durchlaufen werden (vgl. Abb. 4). Auch bei der Verdichtung des Hauptprozesses ,technische Wartung“ konnte in Abhängigkeit von der Beziehung zum physischen Produkt die Unterteilung nach Teilprozessen ersten bis dritten Grades wieder aufgenommen werden. Im Folgenden wird die Kalkulation für einen Wartungsauftrag an einer Maschine dargestellt, bei der zehn Futter der Klasse 1 montiert sind und diese Futter jährlich (also insgesamt dreimal) gewartet werden müssen.

Der Mittelwert (,„Medium“) für die einzelnen Teilprozesse und Hauptprozesskosten resultiert dabei aus den Kostenstelleninformationen. (So ergeben sich beispielsweise die Teilprozesskosten der Demontage von 288,5€ aus dem Teilprozesskostensatz in Abbildung 42 Klasse 1 multipliziert mit der Anzahl von 10 Futtern der Klasse 1, die demontiert werden müssen. Gleichzeitig können auch Vielfache dieser Anzahl von Futtern für andere Kunden ohne Probleme berücksichtigt und geplant werden.)

Das Vorgehen verdeutlicht beispielhaft die Grundlage für eine Angebotskalkulation für einen Standardwartungsvertrag. Durch eine Multiplikation der einzelnen Teilprozesssätze mit den geplanten Kostentreibermengen lassen sich die einzelnen auftragsbezogenen Kosten erheben. Dabei erfolgte kostentreiberabhängig eine Planung der Kosten für den drei Jahre dauernden Standardwartungsvertrag der Geräte GmbH. Teilprozesse, die eine „Anzahl der Wartung“, „Anzahl der Anrufe“ oder ,km Entfernung“ als Kostentreiber haben, wurden so dreimalig (je 
einmal jährlich) in den Kosten des Auftrages berücksichtigt. Die Teilprozesse mit dem Kostentreiber „Anzahl Auftrag“ waren hingegen nicht von der Laufzeit abhängig. Mit dieser Vorgehensweise konnte die Genauigkeit der Planung bzw. die tatsächlichen Planungsunsicherheiten durch die Schwankungsbreiten messbar gemacht werden.

\section{Fazit}

„Leistungsfähigeren Ansätzen des Dienstleistungscontrolling kommt in der Zukunft entscheidende Bedeutung zu." (Meffert u. Bruhn 2003, S. 744). Im vorliegenden Beitrag wurde ein solcher Bestandteil aufgezeigt: Die prozessorientierte Planung und Kalkulation für (kern-)produktbegleitende Dienstleistungen. Dazu wurde eine fünfphasige Vorgehensweise entwickelt und anhand einer praktischen Anwendung dargestellt. In der Praxisanwendung konnte gezeigt werden, dass die Vorgehensweise die systematische Planung von Kosten und damit die Kalkulation von (kern-)produktbegleitenden Dienstleistungen ermöglicht bzw. erheblich verbessert. Daneben unterstützt das fünfphasige Konzept die gezielte, strukturierte und kostenorientierte Entwicklung von (kern-)produktbegleitenden Dienstleistungen.

Die Konzeption erscheint vor allem für Dienstleistungen relevant, bei denen eine starke Beziehung zum physischen Produkt besteht, da dann eine analytische Prozessplanung deutlich einfacher ist. Weniger geeignet und damit von geringerer Relevanz ist die Konzeption daher für ,,produktferne“ Dienstleistungen wie Finanzierung. Spezielle Limitationen bezüglich der Genauigkeit der internen Kapazitäts- und Kostenplanung ergeben sich aus dem Einsatz von Schätzungen zur Bestimmung von Teilprozesszeiten und Schwankungsbreiten. Komplexitätsbedingte Grenzen der Prozesskostenplanung konnten durch die Einbindung eines Time-Driven Activity-Based Costing reduziert werden.

Eine Ausweitung auch auf Zulieferer und Entwicklungspartner der physischen Produkte erscheint vielversprechend. Dies gilt besonders durch die Zunahme und steigende Bedeutung der kooperativen Leistungserstellung generell und in Forschung und Entwicklung speziell. Eine Herausforderung besteht aus der Praxissicht weiterhin in einer IT-technischen Einbindung der Planung und Kalkulation der Dienstleistungen. Häufig sind die IT-Systeme noch nicht für die Einbindung der Dienstleistungen ausgelegt.

Schließlich besteht ein wesentlicher inhaltlicher Forschungsbedarf an der Schnittstelle zum Marketing. Besonders die Integration der prozessorientierten Kostenplanung im Bereich des Preismanagements erscheint hohes Forschungspotenzial zu besitzen. ${ }^{41}$ Auch auf diesem Forschungsfeld kann das vorgestellte Vorgehen als Ansatz dienen.

Danksagung Die Verfasser danken zwei anonymen Gutachtern für wertvolle Hinweise zu der eingereichten Version des Aufsatzes.

\footnotetext{
${ }^{41}$ So existieren bereits Veröffentlichungen zur Preisdifferenzierung von Dienstleistungen. Vgl. exemplarisch Skiera (1999) sowie zur Preiswahrnehmung von Dienstleistungen Siems (2003).
} 
Open Access Dieser Artikel wird zu den Bedingungen der „Creative Commons Attribution Noncommercial License“ zur Verfügung gestellt. Damit ist eine nichtkommerzielle Nutzung, Verbreitung und Vervielfältigung erlaubt, sofern die Autoren des Artikels und die genaue Quelle angegeben sind.

\section{Literatur}

Barth, T.: Outsourcing unternehmensnaher Dienstleistungen: Ein konfigurierbares Modell für die optimierte Gestaltung der Wertschöpfungstiefe. Peter Lang Verlag, Frankfurt am Main (2003)

Belz, C.: Industrieunternehmen als internationale Dienstleister. In: Bieger, T. (Hrsg.) Dienstleistungsmanagement, 2. Aufl., S. 430-453. Paul Haupt Verlag, Bern et al. (2000)

Biermann, T.: Dienstleistungs-Management. Hanser Fachbuch, München (1999)

Borrmann, A.: Service-Controlling für produzierende Unternehmen. Shaker, Aachen (2003)

Bruggeman, W., Moreels, K.: Time-driven activity-based costing - a new paradigm in cost management. In: Horváth, P. (Hrsg.) Performancesteigerung und Kostenoptimierung: Neue Wege und erfolgreiche Praxislösungen, S. 51-66. Schäffer-Poeschel Verlag, Stuttgart (2003)

Bruhn, M.: Qualitätsmanagement für Dienstleistungen: Grundlagen, Konzepte, Methoden. Springer Verlag, Berlin (2004)

Bruhn, M., Meffert, H. (Hrsg.): Handbuch Dienstleistungsmanagement: Von der strategischen Konzeption bis zur praktischen Umsetzung. Dr. Th. Gabler Verlag, Wiesbaden (2001)

Bullinger, H.-J., Meiren, T.: Service Engineering - Entwicklung und Gestaltung von Dienstleistungen. In: Bruhn, M., Meffert, H. (Hrsg.) Handbuch Dienstleistungsmanagement, S. 149-175. Dr. Th. Gabler Verlag, Wiesbaden (2001)

Burr, W.: Service Engineering bei technischen Dienstleistungen - Eine ökonomische Analyse der Modularisierung, Leistungstiefengestaltung und Systembündelung. Deutscher Universitäts-Verlag, Wiesbaden (2002)

Buttler, G., Stegner, E.: Industrielle Dienstleistungen. ZfbF 42(11), 931-946 (1990)

Cibis, C., Niemand, S.: Planung und Steuerung funktioneller Dienstleistungen mit Target Costing dargestellt am Beispiel der IBM Deutschland GmbH. In: Horváth, P. (Hrsg.) Marktnähe und Kosteneffizienz schaffen, S. 191-228. Schäffer-Poeschel Verlag, Stuttgart (1993)

Coners, A., von der Hardt, G.: Time-driven activity-based costing: Motivation und Anwendungsperspektiven. ZfCM 48(2), 108-119 (2004)

Corsten, H.: Betriebswirtschaftslehre von Dienstleistungsunternehmen. Oldenbourg Verlag, München (1990)

Corsten, H.: Dienstleistungsmanagement. Oldenbourg Verlag, München (2001)

Corsten, H., Gössinger, R.: Konzeptionelle und methodische Überlegungen zu einem Controlling für Dienstleistungen. Schriften zum Produktionsmanagement, Nr. 54. Kaiserslautern (2003)

Cooper, R., Kaplan, R.S.: The Design of Cost Management Systems. Prentice-Hall, Upper Saddle River (1999)

Cooper, R., Slagmulder, R.: Strategic cost management: expanding scope and boundaries. J. Cost Manag. 17(1/2), 23-30 (2003)

Downar, P.: Vermarktung industrieller Dienstleistungen: Strategien, Prozesse, Strukturen. Deutscher Universitäts-Verlag, Wiesbaden (2003)

Engelhardt, W.H.: Einige Anmerkungen zur Weiterführung der Diskussion um das Dienstleistungsmarketing. Unternehmung 56(1), 47-50 (2002)

Engelhardt, W.H., Kleinaltenkamp, M., Reckenfelderbäumer, M.: Leistungsbündel als Absatzobjekte. $\mathrm{ZfbF}$ 45(5), 395-426 (1993)

Fischer, R.: Dienstleistungs-Controlling: Grundlagen und Anwendungen. Dr. Th. Gabler Verlag, Wiesbaden (2000)

Fitzsimmons, J.A., Fitzsimmons, M.J.: Service Management: Operations, Strategy and Information Technology, 3. Aufl. McGraw-Hill Professional, Boston (2000)

Franz, K.P.: Die Prozesskostenrechung. Wirtschaftswissenschaftliches Stud. 21(3), 605-610 (1991)

Frehse, J.: Internationale Dienstleistungskompetenzen: Erfolgsstrategien für die europäische Hotellerie. Gabler Verlag, Wiesbaden (2002)

Glaser, H.: Prozesskostenrechnung: Darstellung und Kritik. ZfbF 44(3), 276 (1992)

Göbl, M.: Die Beurteilung von Dienstleistungen. Gabler Verlag, Wiesbaden (2003)

Gouthier, M.H.J.: Kundenentwicklung im Dienstleistungsbereich. Gabler Verlag, Wiesbaden (2003) 
Hope, C., Mühlemann, A.: Service Operations Management - Strategy, Design, Delivery. Prentice-Hall, London (1997)

Horváth, P.: Controlling. Vahlen Verlag, München (2006)

Horváth, P., Mayer, R.: Prozeßkostenrechnung. Der neue Weg zu mehr Kostentransparenz und wirkungsvolleren Unternehmensstrategien. Controlling 1(4), 214-219 (1989)

Horváth, P., Mayer, R.: Prozeßkostenrechnung - Konzeption und Entwicklungen. Kostenrechnungspraxis 37(Sonderheft 2), 15-28 (1993)

Kaplan, R.S., Anderson, S.R.: Time-driven activity-based costing. Harv. Bus. Rev. 82(11), 131-138 (2004)

Kaplan, R.S., Anderson, S.R.: Time-Driven Activity-Based Costing. McGraw-Hill Professional, Boston (2007)

Kaplan, R.S., Cooper, R.: Cost \& Effect: Using Integrated Cost Systems to Profitability and Performance. McGraw-Hill Professional, Boston (1998)

Kersten, W., Zink, T., Kern, E.-M.: Wertschöpfungsnetzwerke zur Entwicklung und Produktion hybrider Produkte: Ansatzpunkte und Forschungsbedarf. In: Blecker, T., Gemünden, H.G. (Hrsg.) Wertschöpfungsnetzwerke, S. 189-202. Schmidt Verlag, Wiesbaden (2006)

Killinger, S.: Produktbegleitende Dienstleistungen. In: Corsten, H., Schneider, H. (Hrsg.) Wettbewerbsfaktor Dienstleistung: Produktion von Dienstleistungen - Produktion als Dienstleistung, S. 129155. Vahlen Verlag, München (1999)

Kinkel, S.: Produktbegleitende Dienstleistungen - Herausforderung für das Controlling. In: Kinkel, S., Jung Erceg, P., Lay, G. (Hrsg.) Controlling produktbegleitender Dienstleistungen - Methoden und Praxisbeispiele zur Kosten- und Erlössteuerung, S. 1-12. Physica Verlag, Heidelberg (2003)

Kleinaltenkamp, M.: Begriffsabgrenzungen und Erscheinungsformen von Dienstleistungen. In: Bruhn, M., Meffert, H. (Hrsg.) Handbuch Dienstleistungsmanagement, S. 27-50. Dr. Th. Gabler Verlag, Wiesbaden (2001)

Korell, M., Ganz, W.: Design hybrider Produkte - Der Weg vom Produkthersteller zum Problemlöser. In: Bullinger, H.J. (Hrsg.) Wettbewerbsfaktor Kreativität: Strategien, Konzepte und Werkzeuge zur Steigerung der Dienstleistungsperformance, S. 153-159. Dr. Th. Gabler Verlag, Wiesbaden (2000)

Küpper, H.-U.: Entwicklungslinien der Kostenrechnung in Dienstleistungsunternehmen. In: Scheer, A.W. (Hrsg.), Grenzplankostenrechnung: Stand und aktuelle Probleme, S. 53-82. Gabler Verlag, Wiesbaden (1991)

Luczak, H., Hoeth, U.: Dienstleistungs-Assessment mit ServAs. In: Hansen, W., Kamiske, G.F. (Hrsg.) Qualitätsmanagement im Dienstleistungsbereich, S. 109-129. Symposion Publishing, Düsseldorf (2003)

Mayer, R.: Kapazitätskostenrechnung. Vahlen Verlag, München (1998)

Meffert, H.: Zukünftige Forschungsfelder im Dienstleistungsmarketing. Unternehmung 55(1), 327-339 (2001)

Meffert, H., Bruhn, M.: Dienstleistungsmarketing: Grundlagen - Konzepte - Methoden. Gabler Verlag, Wiesbaden (2003)

Meyer, A.: Dienstleistungs-Marketing. Betriebswirtschaft 51(2), 195-209 (1991)

Meyer, A.: Dienstleistungs-Marketing: Grundlagen und Gliederung des Handbuches. In: Meyer, A. (Hrsg.) Handbuch Dienstleistungs-Marketing, S. 3-22. Schäffer-Poeschel Verlag, Stuttgart (1998a)

Meyer, A.: Perspektiven im Dienstleistungs-Marketing. In: Meyer, A. (Hrsg.) Handbuch DienstleistungsMarketing, S. 190-197. Schäffer-Poeschel Verlag, Stuttgart (1998b)

Nemeth, R., Ohlhausen, P.: Praxisbeispiel Siemens Medizintechnik - Erhöhung der Kundenperformance durch die Generierung hybrider Produkte. In: Bullinger (Hrsg.) Wettbewerbsfaktor Kreativität: Strategien, Konzepte und Werkzeuge zur Steigerung der Dienstleistungsperformance, S. 171-177. Dr. Th. Gabler Verlag, Wiesbaden (2000)

Niemand, S.: Target Costing für industrielle Dienstleistungen. Vahlen Verlag, München (1996)

Norman, R.: Service Management: Strategy and Leadership in Service Business. Wiley, Chichester et al. (2000)

Olemotz, T.: Strategische Wettbewerbsvorteile durch industrielle Dienstleistungen. Peter Lang Verlag, Frankfurt am Main (1995)

Pak, H., Phipps, C. Spathis, T.: Managing a sprawling service business. The McKinsey Quarterly 38, 79-88 (2002). (Special Edition)

Palloks-Kahlen, M., Kuczynski, S.: Controlling im Service-Management. In: Controlling 12, 135-144 (2000) 
Paul, M., Reckenfelderbäumer, M.: Preisbildung und Kostenrechnung auf der Basis neuer Kostenrechnungsverfahren. In: Bruhn, M., Meffert, H. (Hrsg.) Handbuch Dienstleistungsmanagement, S. 627-659. Dr. Th. Gabler Verlag, Wiesbaden (2001)

Pepels, W.: Qualitätscontrolling bei Dienstleistungen. Vahlen Verlag, München (1996)

Pepels, W.: Dienstleistungen. Wirtschaftsstudium 33(7), 883-886 (2004)

Reckenfelderbäumer, M.: Marketing-Accounting im Dienstleistungsbereich - Konzeption eines prozeßkostengestützten Instrumentariums. Dr. Th. Gabler Verlag, Wiesbaden (1995)

Reckenfelderbäumer, M.: Entwicklungsstand und Perspektiven der Prozesskostenrechnung. Gabler Verlag, Wiesbaden (1998)

Reckenfelderbäumer, M.: Ausblick: Die Kalkulation von Betreibermodellen als zukünftige Herausforderung für das Controlling produktbegleitender Dienstleistungen. In: Kinkel, S., Jung Erceg, P., Lay, G. (Hrsg.) Controlling produktbegleitender Dienstleistungen - Methoden und Praxisbeispiele zur Kosten- und Erlössteuerung, S. 169-186. Physica Verlag, Heidelberg (2003)

Reckenfelderbäumer, M.: Die Wirtschaftlichkeitsanalyse von dienstleistungsorientierten Geschäftsmodellen als Herausforderung an das Controlling. In: Meier, H. (Hrsg.) Dienstleistungsorientierte Geschäftsmodelle im Maschinen- und Anlagenbau - Vom Basisangebot bis zum Betreibermodell, S. 209-241. Springer Verlag, Berlin (2004)

Reichwald, R., Goecke, R., Stein, S.: Dienstleistungsengineering - Dienstleistungsvernetzung in Zukunftsmärkten. TcW, München (2000)

Reiß, M., Präuer, A.: Solutions providing - Was ist Vision - was Wirklichkeit? Absatzwirtschaft 44(7), 48-53 (2001)

Rösner, J.: Service - ein strategischer Erfolgsfaktor von Industrieunternehmen. Schmidt Verlag, Hamburg (1998)

Roth, S.: Preismanagement für Leistungsbündel. Gabler Verlag, Wiesbaden (2006)

Rück, H.R.G.: Dienstleistungen in der ökonomischen Theorie. Deutscher Universitäts-Verlag, Wiesbaden (2000)

Schneider, R.: Produktbegleitende Dienstleistung. Wissenschaftsmanagement 4(6), 10-13 (1998)

Schuh, G., Fridli, T., Gebauer, H.: Fit for Service: Industrie als Dienstleister. Hanser Wirtschaft, München (2004)

Schuh, G., Speth, H.: Controlling industrieller Dienstleistungen. io Manag. 68(11), 32-39 (1999)

Schüller, A.: Dienstleistungsmärkte in der Bundesrepublik Deutschland: Sichere Domänen selbständiger mittelständischer Unternehmen? Westdeutscher Verlag, Köln (1967)

Schweitzer, M., Küpper, H.-U.: Systeme der Kosten- und Erlösrechnung. Vahlen Verlag, München (2003)

Schwengels, C.: Kostenorientierte Entwicklung von Dienstleistungen. In: Bullinger, H.-J., Scheer, A.-W. (Hrsg.) Service Engineering: Entwicklung und Gestaltung innovativer Dienstleistungen, S. 507529. Springer Verlag, Berlin (2003)

Serfling, K., Jeiter, V.: Gemeinkostencontrolling in Dienstleistungsbetrieben auf Basis der Prozesskostenrechnung. Kostenrechnungspraxis 39(6), 321-329 (1995)

Shostak, G.L.: Designing services that deliver. Harv. Bus. Rev. 62(1/2), 133-139 (1984)

Siems, F.: Preiswahrnehmung von Dienstleistungen - Konzeptualisierung und Integration in das Relationship Marketing. Gabler Verlag, Wiesbaden (2003)

Simon, H.: Industrielle Dienstleistungen und Wettbewerbsstrategie. In: Simon, H. (Hrsg.) Industrielle Dienstleistungen, S. 3-22. Schäffer-Poeschel Verlag, Stuttgart (1993)

Skiera, B.: Mengenbezogene Preisdifferenzierung bei Dienstleistungen. Wiesbaden (1999)

Spath, D., Demuß, L.: Entwicklung hybrider Produkte - Gestaltung materieller und immaterieller Leistungsbündel. In: Bullinger, H.-J., Scheer, A.-W. (Hrsg.) Service Engineering: Entwicklung und Gestaltung innovativer Dienstleistungen, S. 467-506. Springer Verlag, Berlin (2003)

Spath, D., Zahn, E. (Hrsg.) Kundenorientierte Dienstleistungsentwicklung in deutschen Unternehmen. Berlin (2003)

Spath, D., Ganz, W., Schreiner, P.: Kundenorientiertes Dienstleistungsmanagement als Gestaltungsaufgabe für die Unternehmensführung. In: Spath, D. (Hrsg.) Forschungs- und Technologiemanagement: Potenziale nutzen - Zukunft gestalten, S. 281-287. Hanser Fachbuchverlag, München (2004)

Stanik, M.: Customer related service engineering - Wettbewerbsvorteile durch kundenorientiertes Service Engineering. In: Zahn, E., Spath, D., Scheer, A.-W. (Hrsg.) Vom Kunden zur Dienstleistung: Methoden, Instrumente und Strategien zum Customer related Service Engineering, S. 16-24. IRB Verlag, Stuttgart (2004)

Stauss, B., Bruhn, M.: Dienstleistungsnetzwerke - Eine Einführung in den Sammelband. In: Bruhn, M., Stauss, B. (Hrsg.) Dienstleistungsmanagement Jahrbuch 2003: Dienstleistungsnetzwerke, S. 3-30. Gabler Verlag, Wiesbaden (2003) 
Statistisches Bundesamt: http://www.destatis.de Zugriff am 21.1.2008

Steven, M., Schade, S.: Produktionswirtschaftliche Analyse industrieller Dienstleistungen. Z. Betriebswirtsch. 74, 543-562 (2004)

Stoi, R.: Prozessorientiertes Kostenmanagement in der deutschen Unternehmenspraxis. Vahlen Verlag, München (1999)

Storey, C., Kelly, D.: Measuring the Performance of new service development activities. Service Industries J. 21(2), 71-90 (2001)

Thomke, S.: R\&D comes to service. Harv. Bus. Rev. 81(4), 71-79 (2003)

VDI: VDI-Richtlinie 2221: Methodik zum Entwickeln und Konstruieren technischer Systeme und Produkte. VDI, Düsseldorf (1993)

Vikas, K.: Grenzplankostenrechnung im Dienstleistungsbereich. In: Scheer, A.-W. (Hrsg.) Grenzplankostenrechnung: Stand und aktuelle Probleme, S. 229-259. Gabler Verlag, Wiesbaden (1991)

Weber, R.: Kostenmanagement für Dienstleister und Non-Profit-Unternehmen. Expert Verlag, RenningenMalmsheim (1999)

Weber, J.: Editorial: Dienstleistungs-Controlling. Kostenrechnungspraxis 46(2), 1 (2002). (Sonderheft)

Weber, J., Schäffer, U.: Controlling in Dienstleistungsunternehmen. In: Bruhn, M., Meffert, H. (Hrsg.) Handbuch Dienstleistungsmanagement, S. 899-913. Dr. Th. Gabler Verlag, Wiesbaden (2001)

Witt, F.-J.: Dienstleistungscontrolling. Vahlen Verlag, München (2003)

Woratschek, H.: Zum Stand einer ,,Theorie des Dienstleistungsmarketing“. Die Unternehmung 55(4/5), 261-278 (2001a)

Woratschek, H.: Preisbildung im Dienstleistungsbereich auf der Basis von Marktinformationen. In: Bruhn, M., Meffert, H. (Hrsg.) Handbuch Dienstleistungsmanagement, S. 606-625. Wiesbaden (2001b) 